\title{
Du bon usage de la parenté construite avec des humeurs corporelles (sang et lait) et quelques autres moyens
}

of the proper use of kinship made from bodily humors (blood and milk) and some other means

\section{Bernard Vernier}

\section{(2) OpenEdition}

\section{Journals}

\section{Édition électronique}

URL : http://journals.openedition.org/ejts/623

DOI : $10.4000 /$ ejts. 623

ISSN : 1773-0546

Éditeur

EJTS

\section{Référence électronique}

Bernard Vernier, «Du bon usage de la parenté construite avec des humeurs corporelles (sang et lait) et quelques autres moyens », European Journal of Turkish Studies [En ligne], 4 | 2006, mis en ligne le 04 mars 2015, consulté le 16 février 2020. URL : http://journals.openedition.org/ejts/623 ; DOI : 10.4000/ ejts. 623 


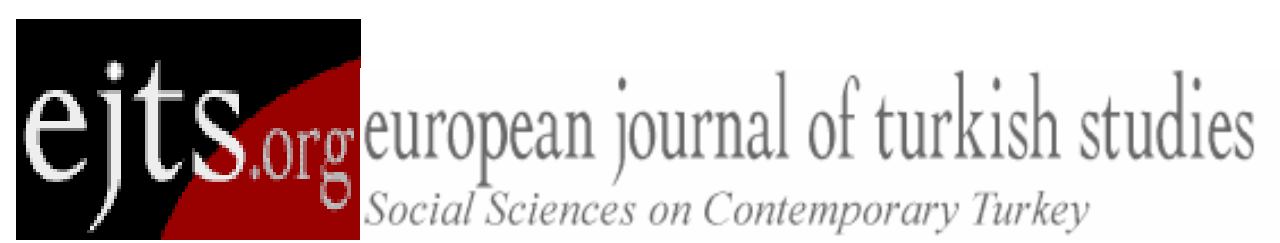

Citation: Vernier, Bernard (2006) 'Du bon usage de la parenté construite avec des humeurs corporelles (sang et lait) et quelques autres moyens', European Journal of Turkish Studies, Thematic Issue $\mathrm{N}^{\circ} 4$, The social practices of kinship. A comparative perspective, URL : http://www.ejts.org/document623.html

To quote a passage, use paragraph (§).

\title{
Du bon usage de la parenté construite avec des humeurs corporelles (sang et lait) et quelques autres moyens
}

\author{
Bernard Vernier
}

\begin{abstract}
Family tie is so important that it has often to be invented when it does not exist. Many ties (by blood, by milk etc.) which can be substitutive to the family tie and which can be limitless in accordance with the needs of social context, allow to extend the advantages of mutual assistance or of good functioning of the hierarchical relations. Each of one of these ties in its own fashion, which is often accompanied by an interdiction of incest, endeavors to compensate the lack of ordinary ties, to protect against the concealed threats and to regulate the functioning of ordinary ties. They can even compete with the ordinary ties. We insist here on their common characteristic. Each follows a specific logic that remains largely to be explored. It is necessary to consider their support but also one should avoid the trap of substantialism, which neglects the capacity of societies to pretend.
\end{abstract}



quelques autres moyens', European Journal of Turkish Studies, Thematic Issue $\mathrm{N}^{\circ} 4$, The social practices of kinship. A comparative perspective, URL : http://www.ejts.org/document623.html

To quote a passage, use paragraph $(\S)$

La parenté 'consanguine' a, surtout en l'absence d'Etat ou d'autorité centralisée, une importance vitale pour la survie. La parenté par alliance matrimoniale améliore les chances de survie en redoublant et donc en renforçant certains liens de parenté ou, surtout, en étendant le champ de la parenté à des individus, des familles ou des groupes (groupes de parenté ou classes matrimoniales) parfois très importants numériquement. Elle permet alors de traiter des non parents comme de quasi parents et d'obtenir d'eux un ensemble de services qui dépendent dans chaque cas de la société considérée et qui sont souvent, pour une part importante, distincts de ceux qu'on peut attendre des parents ordinaires. En ce sens, la parenté par alliance matrimoniale est complémentaire de la parenté consanguine. Mais elle a elle-même ses limites. Les hommes ont souvent eu besoin de se lier par des relations stables (à usages multiples) avec des individus, des familles, lignages, clans, tribus avec qui ils ne pouvaient ou ne voulaient pas se marier. II est, bien sûr, toutes sortes de façon de créer des relations stables, mais les relations de parenté constituent, dans certaines conditions d'existence en voie de disparition, un des moyens les plus efficaces. La parenté est souvent si importante qu'il faut l'inventer quand elle n'existe pas. Les hommes ont inventé une multitude de formes d'alliance différentes de l'alliance matrimoniale : parenté de baptême, de circoncision, de la première coupe des cheveux, homonymique, etc. Chacune d'entre elles a certes sa spécificité, mais elles ont en commun de construire de la parenté à des fins intéressées. Pour des raisons pratiques, je me contenterai de présenter de façon schématique deux formes de ces parentés construites (au même titre que l'alliance matrimoniale) : la parenté de sang et la parenté de lait. Pourquoi celles-là? Le parrainage est déjà bien connu. Je n'en parlerai que dans la conclusion à des fins comparatives. Pour la parenté de sang, j'ai eu la chance de pouvoir en observer moi-même l'usage au Nigéria. Surtout, en étudiant les parentés fondées sur l'utilisation des humeurs corporelles on se donne, du même coup, le moyen d'évaluer la pertinence d'une interprétation substantialiste de la parenté. J'ai choisi de donner une grande importance aux études de cas pour que le lecteur puisse vérifier par luimême la pertinence des conclusions généralisantes que je présente dans la conclusion. Pour chaque auteur cité je me suis efforcé de montrer l'originalité de son étude du point de vue théorique, du point de vue de ou des approches utilisée(s) - anthropologie sociale et /ou du symbolique, etc. - ou tout simplement sous le rapport des thèmes abordés. 

quelques autres moyens', European Journal of Turkish Studies, Thematic Issue $\mathrm{N}^{\circ} 4$, The social practices of kinship. A comparative perspective, URL : http://www.ejts.org/document623.html

To quote a passage, use paragraph $(\S)$

\section{La parenté par le sang}

[2] Avant d'analyser un cas nigérian je signalerai, rapidement, quelques anciens travaux sur la fraternité de sang qui, à des titres divers, me semblent importants. Tous les exemples analysés seront africains. Mais pour avoir une idée complète de l'importance de cette institution il faut savoir que l'alliance par le sang était très répandue depuis l'antiquité. L'historien grec Hérodote (I, 74, III 8 , IV 70.) raconte que les Scythes exécutaient le pacte de sang en pratiquant une incision à l'aide d'un couteau ou d'un glaive et en mélangeant les sangs à du vin dans une coupe. Ils trempaient ensuite leurs armes (épées, flèches, javelots, etc.) dans celle-ci, prononçaient une longue malédiction à l'adresse des parjures, puis les chefs et leurs partisans de haut rang buvaient le mélange de sang et de vin. II mentionne aussi le cas des Mèdes et des Lydiens qui se faisaient des incisions au bras et se léchaient le sang et celui d'un pacte arabe au cours duquel les contractants se tiraient mutuellement du sang et le répandaient sur sept pierres placées entre eux. Pour certaines sociétés musulmanes en tout cas on sait (Tegnaeus 1952) que les femmes musulmanes ne se voilaient pas en présence de frères de sang (même s'il s'agissait de chrétiens) traités donc comme s'ils étaient réellement des frères avec qui il était de toute façon interdit d'avoir des rapports sexuels. La fraternité de sang existait aussi chez les Grecs, les Romains et dans toute l'Europe jusqu'en Scandinavie et elle était utilisée en Albanie pour arrêter les vendettas devenues impossibles entre frères de sang (Durham 1909).

[3] L'expression 'fraternité de sang' qu'on utilise par commodité est impropre. Parfois l'échange de sang est remplacé (Tegnaeus 1952) en Afrique par un échange de chair, de salive (Somalis et Gallas) ou par l'absorption d'une boisson dans laquelle on a délayé un peu de la terre du lieu (Yourouba). Chez les Karen de Birmanie un repas pris en commun permet la cessation des hostilités pour une période limitée et la plantation d'un arbre, pour la durée de vie de l'arbre. Parfois le sang n'est pas absorbé mais répandu dans le cadre d'un sacrifice. C'est souvent le cas en pays musulman où la fraternité de sang a été interdite car le sang ne doit couler que pour Dieu. Jaussen (1908) raconte qu'au pays de Moab deux hommes mariés ou célibataires peuvent décider de 'réunir leurs effort dans une vie commune. Ils convoquent un certain nombre de témoins et en leur présence ils immolent une victime appelée (..) victime du ruag (ou de la tente). En répandant le sang, ils disent: 
Citation: Vernier, Bernard (2006) 'Du bon usage de la parenté construite avec des humeurs corporelles (sang et lait) et quelques autres moyens', European Journal of Turkish Studies, Thematic Issue $\mathrm{N}^{\circ} 4$, The social practices of kinship. A comparative perspective, URL : http://www.ejts.org/document623.html

To quote a passage, use paragraph $(\S)$

'par la vertu de cette dahiheh nous sommes devenus une seule famille'. Ils mettent désormais 'tout (..) en commun : habitation, nourriture, travail, gain et perte'. Jaussen raconte aussi qu'il existait dans cette société une forme d'adoption (sans droit d'héritage) qui était consacrée, après un séjour et un travail de plusieurs années dans une famille, par le sacrifice d'une victime 'à la face d'Allah'. Le père prononçait les paroles d'adoption et désormais la personne portait son nom et était censée 'participer à son sang' (Jaussen 1908: 25). Au mariage mari et femme étaient aussi liés par le sang. Le mariage s'accompagnait par un sacrifice mais pour mieux sceller l'alliance le mari mettait du sang sur le front et la poitrine de sa femme et dans certains groupes il s'en enduisait aussi une partie du corps (Jaussen 1908: 54).

[4] Dans la plupart des cas, semble-t-il, la transgression d'un pacte de paix basé sur l'échange ou l'absorption de substances comporte des sanctions automatiques (parce que magicoreligieuses) très graves.

\section{Sur quelques travaux pionniers}

[5] Henri Labouret (1929) observait dans un travail de synthèse qu'il existe en Afrique des rapports de parenté à plaisanterie non seulement entre parents ou entre alliés (cousins croisés, oncle/ neveu, grands-parents /petits-enfants, beaux-frères/belles sœurs, etc.) mais aussi, comme au Soudan (chez les Manding les Peuls et les Toucouleurs) et en Haute Volta (Dian, Lobi), entre clans d'une même tribu, entre une population et une catégorie professionnelle (Peuls avec les forgerons) entre familles différentes, entre villages, régions, et même peuples différents. II notait que cette parenté à plaisanterie pouvait être établie par un échange de sang et soulignait qu'elle pouvait lier des groupes égaux ou inégaux.

[6] Chez les Wolof les clans qui s'insultent forment entre eux un rapport maître (sange) et esclave (dyam). Mais chaque famille possède ses esclaves (dyam) souvent descendants d'ancêtres avec qui la famille a eu la même relation : l'échange de services et de cadeaux ne se fait donc en pratique qu'entre quelques uns. Dans les grandes fêtes musulmanes l'esclave aide son maître (participe à la cuisine etc.) et la famille de celui-ci lui donne en retour nourriture et cadeaux. Au mariage, l'esclave intervient dans les négociations entre les familles (dot, travail que le gendre doit à son beau-père, etc.). II recrute de la main-d'œuvre pour travailler la terre de son beau-père et 
Citation: Vernier, Bernard (2006) 'Du bon usage de la parenté construite avec des humeurs corporelles (sang et lait) et quelques autres moyens', European Journal of Turkish Studies, Thematic Issue $N^{\circ} 4$, The social practices of kinship. A comparative perspective, URL : http://www.ejts.org/document623.html

To quote a passage, use paragraph (§)

s'engage dans une compétition avec les travailleurs recrutés par le gendre. C'est un esclave qui dépose la fiancée sur sa couche et qui intervient plus tard au moment de l'accouchement. II intervient comme médiateur dans les disputes entre les maîtres (contestations de propriété, rixe entre jeunes). II préside à la récolte, discute des conditions de la vente et surveille les pesées. II s'occupe du cadavre de son maître, le lave, l'habille, organise le repas et préside au partage des affaires du mort (bestiaux, argent, parures etc.). Si le mort est une femme c'est l'épouse de l'esclave qui lave la morte, etc. Ajoutons qu'on est obligé de racheter 'l'esclave' qui est devenu un véritable esclave et qu'on ne peut pas témoigner en justice contre son partenaire. Cette relation à plaisanterie maîtres/esclaves existe aussi entre peuples différents. Au Sénégal, les Peuls sont les maîtres et les Serer leurs esclaves. Mais ailleurs les Peuls sont les esclaves des Berbères et des Wangara. De façon générale cette parenté à plaisanterie implique un échange de cadeaux et de services réciproques qui varient selon les cas, ainsi que le droit de s'injurier sans conséquence. Labouret tirait de ces observations une conclusion qui vaut autant pour la fraternité de sang que pour la parenté à plaisanterie (chacune de ces institutions pouvant exister indépendamment de l'autre) et dont il ne faut pas sous-estimer l'importance théorique : en Afrique les populations sont liées par une chaîne de réciprocité beaucoup plus étroite qu'on ne le soupçonne d'ordinaire ${ }^{1}$.

[7] En 1933, Evans-Pritchard publie un article capital sur la fraternité de sang chez les Zandé du sud-ouest du Soudan. II donne une description précise du rite qui l'inaugure, s'interroge sur la signification de l'échange de sang (crée-t-il une identité substantielle entre partenaires, une véritable parenté consanguine?) et analyse les fonctions de cette fraternité en les comparant à celles de la parenté consanguine et par alliance.

[8] Chez les Zandé, la fraternité de sang est un pacte d'alliance formé (après consultation de l'oracle pour être sûr que l'alliance sera bénéfique) entre deux personnes (en général des hommes) par un acte rituel au cours duquel chacun avale le sang de l'autre à partir d'une incision au bras ou à la poitrine. II se crée une 'parenté' entre les familles des frères de sang (la femme du frère de sang devient ma 'sœur de sang') mais aussi, dans une beaucoup moindre mesure (il en parle peu), entre les clans dont ils sont membres.

1 Tagnaeus cite des cas de pactes de sang entre explorateurs (et parfois même missionnaires) blancs et chefs d'Afrique ou d'Asie. 
Citation: Vernier, Bernard (2006) 'Du bon usage de la parenté construite avec des humeurs corporelles (sang et lait) et quelques autres moyens', European Journal of Turkish Studies, Thematic Issue $N^{\circ} 4$, The social practices of kinship. A comparative perspective, URL : http://www.ejts.org/document623.html

To quote a passage, use paragraph (§)

[9] Ces pactes permettent d'institutionnaliser des liens de camaraderie déjà existants en créant des obligations analogues à celles qui existent entre parents, d'organiser des échanges entre personnes dont les productions sont complémentaires (viande contre lances, etc.) et de permettre le commerce au loin avec l'assurance d'un traitement de faveur, y compris avec les pays ennemis. Ego se procure ainsi un accompagnateur qui garantit sa sécurité. Au cours du rite chaque partenaire énumère la liste des obligations et des interdits entre partenaires. II s'adresse à son propre sang présent dans le corps de l'autre et menace le parjure de sanctions prises très au sérieux. Le frère de sang a le devoir de se comporter comme un ami généreux envers son partenaire et de lui donner tout ce qu'il réclame au cours de ses visites (bière, coq, lance, fer, fille à épouser). Si son ami est accusé d'adultère ou de sorcellerie il doit l'aider à rassembler la compensation qui lui permet d'échapper à la mutilation ou à la mort. II a le devoir de lui rendre un certain nombre de services mortuaires comme ouvrir le corps du cadavre (pour que les parents puissent savoir si le mort était un sorcier, ou s'il était faussement accusé de sorcellerie durant sa vie) ou aider à creuser la tombe des membres de sa famille. II lui est interdit de faire du tort à son partenaire en le blessant physiquement, en disant du mal de lui (au chef), en le rendant malade (par sorcellerie) ou en commettant l'adultère avec sa femme. II doit épouser ses querelles, le prévenir des actions entreprises contre lui, le protéger de la vengeance de ses ennemis et même de la justice du chef.

[10] Comment caractériser la nature de cette relation? Les frères de sang ont une relation d'intimité et d'égalité qui s'exprime dans une relation à plaisanterie où l'on se ment et où l'on s'injurie mais pour rire. Le danger d'adultère étant minime, on a libre accès à la maison de l'autre. Les partenaires se font de fréquentes visites, mangent l'un chez l'autre, s'invitent à des parties de bière et reçoivent une part des bêtes tuées. Cette amitié privilégiée ou mieux cette sorte de fraternité est, bien sûr, d'autant plus utile qu'on a de mauvais rapports avec ses propres consanguins et affins.

[11] En désaccord avec Robertson Smith, Frazer et Davy pour qui l'échange de sang transforme les frères de sang en véritables parents dotés d'une substance commune, EvansPritchard reprend à son compte les idées de Westermarck. Le sang échangé 'n'est qu'un véhicule pratique pour la malédiction.': l'élément le plus important c'est cette malédiction conditionnelle avec les imprécations proférées par les partenaires. Le sang devient, par son association au rite, une substance magique. Sa signification sociologique est très différente de celle du sang ordinaire. Le 
Citation: Vernier, Bernard (2006) 'Du bon usage de la parenté construite avec des humeurs corporelles (sang et lait) et quelques autres moyens', European Journal of Turkish Studies, Thematic Issue $\mathrm{N}^{\circ} 4$, The social practices of kinship. A comparative perspective, URL : http://www.ejts.org/document623.html

To quote a passage, use paragraph $(\S)$

sang est censé entendre le discours qu'on lui adresse et de l'estomac de l'homme voit tout ce que celui-ci fait. Si l'homme trahit son frère il se venge sur lui.

[12] L'échange de sang ne crée donc pas une identité substantielle entre les partenaires. Un certain nombre de pactes n'utilisent d'ailleurs pas le sang. C'est l'occasion pour Evans-Pritchard de dénoncer, bien avant Leach et Schneider, l'ethnocentrisme des ethnologues : ' L'idée que les parents et membres d'un même clan sont des personnes de même sang et qu'en buvant le sang de l'autre on devient son parent est une idée de Blanc'.

[13] L'arsenal des arguments mobilisés par Evans-Pritchard pour démontrer que le pacte de sang chez les Zandé ne crée aucune parenté de sang est impressionnant. Le clan constitue, dans cette société, une unité sociale plus que physiologique. Les membres d'un même clan ne se considèrent pas, eux-mêmes, comme ayant un même sang. Et s'il fallait donner une base substantielle à l'unité du groupe il faudrait dire avec eux que 'les membres d'un même clan sont issus de la même semence sexuelle'. D'ailleurs, s'ils associaient la parenté au sang commun leur système de filiation devrait être matrilinéaire puisque, dans cette société, les enfants sont supposés formés du sang de leur mère. Or les Zandé sont patrilinéaires. Certes ils n'échangent pas de sang avec un membre de leur propre clan mais ce n'est pas parce qu'ils se considèrent déjà de même sang, c'est qu'en tant que parents ils sont déjà liés par des liens sociologiques. C'est aussi parce qu'ils sont déjà leurs parents qu'ils n'échangent pas de sang non plus avec des membres de leur famille maternelle ou des clans des grands parents maternels. En opposition directe avec la théorie du clan comme unité physiologique, les sanctions du parjure impliquent également les membres de la parenté maternelle. Et si l'échange de sang créait une identité substantielle, chacun ne devrait-il pas considérer son partenaire comme membre (au sens physiologique ou au moins sociologique du terme) de son clan ? Or, ce n'est jamais le cas : on n'adopte ni le nom, ni les totems de son frère de sang. On ne peut non plus hériter de lui. Surtout, il n'existe pas de prohibition sexuelle et matrimoniale entre les familles des frères de sang. On trouve en effet souhaitable qu'un homme se marie avec la sœur ou la fille de son frère de clan. Dans la même logique, aux fêtes mortuaires l'échange rituel des biens se fait entre, d'une part, les frères de sang et les parents par alliance classés ensemble (comme si les deux types de liens étaient assimilés) et, de l'autre, les parents naturels. 
Citation: Vernier, Bernard (2006) 'Du bon usage de la parenté construite avec des humeurs corporelles (sang et lait) et quelques autres moyens', European Journal of Turkish Studies, Thematic Issue $N^{\circ} 4$, The social practices of kinship. A comparative perspective, URL : http://www.ejts.org/document623.html

To quote a passage, use paragraph (§)

[14] De grandes différences sociologiques opposent d'ailleurs les rapports entre frères de sang (rapports d'égalité et de familiarité) et entre frères réels (rapports hiérarchisés où les cadets doivent respect aux aînés). Et si de nombreuses obligations sont communes, cela ne vient pas d'une assimilation des uns aux autres 'mais du fait qu'il n'y a pas trente-six façons d'aider les autres : les pactes d'assistance mutuelle sont donc forcés de reproduire les modes d'assistance de la parenté'. Et si les 'obligations de la fraternité de sang sont colorées par celles de la parenté et de la famille, c'est que les principales attitudes sociales d'un homme se construisent d'abord envers la parenté et la famille'. On ne change pas de statut familial et clanique. On reste membre de sa propre famille et de son propre clan.

[15] Pour les Zandé celui qui a bu le sang est un ami beaucoup plus cher qu'un frère réel: 'Il ne commettra pas d'adultère avec votre femme, il ne refusera pas de vous aider en cas de besoin tandis que vos frères sont toujours en train d'essayer de corrompre votre femme et d'éviter de vous aider en cas de difficulté'. La fraternité de sang est choisie, ses obligations sont plus précises et plus réciproques : tout parjure est sanctionné magiquement par la mort².

[16] Au total, un homme 'devient votre frère de sang par les liens de la magie qui l'impliquent dans un ensemble de devoirs quelque fois les mêmes mais souvent différents ou même opposées aux devoirs de la parenté et basés sur une sanction magique typique de la malédiction conditionnelle'.

[17] Dans son livre sur le pacte de sang au Dahomey (1937) Paul Hazoumé ajoute d'autres usages de cette institution : pactes d'entraide entre villages en temps de guerre ou entre personnes de statut clairement inégaux (le souverain avec ses espions internes et externes ou ses confidents) et même entre proches parents: des frères et sœurs conjurés contre l'un d'eux qui a usurpé le pouvoir royal. II est clair que ce dernier cas renforce l'idée selon laquelle le pacte de sang n'est pas là pour créer de la parenté de sang. Le sang n'est que le véhicule magique de la sanction. Hazoumé décrit les rites de nombreuses tribus, mentionne les divinités associées au rite et prend en compte le symbolisme des objets utilisés. II donne des exemples de la façon dont des parjures ont été châtiés dans des cas connus, les rites qui leur permettent d'échapper au châtiment et ceux qui permettent de

2 Selon Evans-Pritchard, les Zandé surestiment l'importance des liens de fraternité de sang. II lui semble que les liens de parenté résistent malgré tout mieux aux différences d'intérêt et aux disputes. 
Citation: Vernier, Bernard (2006) 'Du bon usage de la parenté construite avec des humeurs corporelles (sang et lait) et quelques autres moyens', European Journal of Turkish Studies, Thematic Issue $\mathrm{N}^{\circ} 4$, The social practices of kinship. A comparative perspective, URL : http://www.ejts.org/document623.html

To quote a passage, use paragraph $(\S)$

sortir de la relation de fraternité. Enfin il souligne l'extrême importance sociale des pactes dans cette société.

[18] Les Dahomêens ne se confient de secrets, ne se prêtent une assistance mutuelle, ne s'associent pour une affaire importante (entreprise commerciale, confection d'un gri-gri, entente pour vol, assassinat, évasion, assouvissement d'une haine, etc.), ils ne se dévouent, jusqu'à la mort, les uns pour les autres (obligation de libérer un frère de ses chaînes), ne s'occupent des orphelins les uns des autres que s'ils se sont jurés la confiance, la discrétion, la sincérité, la loyauté, le dévouement, en contractant le pacte de sang. Le pacte crée non pas une affection irrésistible (à l'image de certains filtres), mais du fait de l'existence d'une sanction automatique, une confiance illimitée entre les contractants.

[19] L'importance de cette relation est telle que l'on doit sacrifier l'intérêt de ses parents à celui des 'amis-conjurés'. Dans de nombreux cas où les interdits sont mentionnés ils comportent un interdit des relations sexuelles avec la femme et parfois, contrairement à ce qui se passe chez les Zandé, avec la fille du 'frère'.

[20] Denise Paulme (1939) remarque comme Labouret que les alliances de ce type ont souvent pour origine un service rendu important. C'est le cas de l'alliance (Mangu) chez les Dogons, qui s'accompagne comme chez les Zandé d'un rapport de parenté à plaisanterie où se mêlent injures, obscénité et même une certaine forme de brutalité et qui ressemblent à des comportements qu'on a avec certains parents par alliance mais aussi avec certains autres non-parents. II est interdit de se battre pour de vrai entre ce type d'alliés, mais aussi avec un troisième groupe devant un allié, car ils ne doivent pas voir le sang couler de l'allié devant eux. De ce fait, le Mangou est un médiateur privilégié. II suffit qu'il se montre sur le lieu de la dispute pour ramener l'ordre et la réconciliation. En cas d'actes qui souillent le groupe (ex : offense commise envers l'animal totémique), il joue le rôle de purificateur en procédant aux rites prévus. Elle remarque que le pacte s'accompagne parfois d'interdit de rapports sexuels et matrimoniaux bien que les partenaires ne soient en rien assimilés à des consanguins. Le pacte rend l'allié plus sacré encore que les proches. L'alliance est parfois d'ailleurs dirigée contre les consanguins ou contre les membres du groupe totémique. 
Citation: Vernier, Bernard (2006) 'Du bon usage de la parenté construite avec des humeurs corporelles (sang et lait) et quelques autres moyens', European Journal of Turkish Studies, Thematic Issue $N^{\circ} 4$, The social practices of kinship. A comparative perspective, URL : http://www.ejts.org/document623.html

To quote a passage, use paragraph (§)

[21] Marcel Griaule publie en 1948 un article fameux ${ }^{3}$ sur l'alliance cathartique dite mangou entre Dogons et Bozos du Niger. Cette alliance implique des prestations matérielles, morales et religieuses (hospitalité, nourriture) et un rôle de purification par des rites dans les cas graves (impureté liée à la foudre, à la mort ou à la transgression d'un interdit alimentaire) et de façon plus quotidienne par des échanges d'injures entre alliés. Griaule analyse de façon extrêmement précise la notion de personne qui rend nécessaire cette purification et le mécanisme de celle-ci, incompréhensible sans une connaissance de la mythologie. II insiste lui aussi sur le rôle de conciliateur (ici encore il est donc question de rétablissement de l'ordre) du mangou pour ses alliés. Par sa seule présence, il est capable de ramener le calme chez ses alliés qui se disputent entre eux car il est interdit de lui infliger le spectacle du sang d'un allié qui coule. II est sollicité en dernier recours (quand les notables sont impuissants) pour les disputes entre époux. La femme retournée dans sa famille qui refuse de reprendre sa place au foyer ne peut refuser de suivre le mangou qui vient la chercher. Elle est obligée de reprendre la vie commune, ne serait-ce qu'une journée.

[22] Ces obligations s'accompagnent de l'interdiction absolue de tout rapport sexuel entre les deux peuples et de tout acte comportant versement de sang ou atteinte à la vie. La rupture de tels interdits entraîne une impureté presque impossible à purifier comme le montre l'exemple qui suit. On rapporte qu'un bozo a été tué par des dogons. Quand les coupables se sont aperçus de leur méprise, ils ont abandonné leur village et leurs champs car cette communauté coupable, après un tel crime collectif, ne pouvait plus continuer en tant que telle : ses membres, ses autels, ses eaux étaient devenus impurs.

[23] Ce type d'alliance 'offre de tels avantages, matériels, sociaux, spirituels qu'il sert de modèle pour les alliances que les dogons multiplient à l'infini (...)'. Ils ont tressé un réseau serré d'alliances intérieures dont toutes sont, à des degrés divers, un rappel du pacte bozo : alliances entre groupes, entre régions, entre villages et entre familles d'un même village. Dans chaque cas une légende d'origine fait état, le plus souvent, d'un service rendu qui a donné lieu à un échange de serment fréquemment appuyé par un échange de sang.

[24] L'intérêt du livre de Tegnaeus (1952) sur La fraternité de sang est d'être un travail de synthèse qui apporte des exemples du monde entier tout en restant centré sur l'Afrique. Le pacte de 
Citation: Vernier, Bernard (2006) 'Du bon usage de la parenté construite avec des humeurs corporelles (sang et lait) et quelques autres moyens', European Journal of Turkish Studies, Thematic Issue $N^{\circ} 4$, The social practices of kinship. A comparative perspective, URL : http://www.ejts.org/document623.html

To quote a passage, use paragraph (§)

sang peut lier des personnes, parfois même des parents ou des conjoints ${ }^{4}$ ou des groupes (royaumes, villages, tribus, clans, familles, personnes de religion différentes). Le plus souvent il interdisait le mariage avec les membres du groupe allié ou avec les proches parentes du frère (chez les Zoulou, Kaonde, Gandas, Acolis, Dinka, Tanala, Dogon) mais parfois non (chez les Turcs de Bulgarie orientale, les Zandé et les Kaguru). II arrive même alors que les amis se prêtent leur femme (Bantous, Nyoro) à l'exception, dans certaines sociétés (Mbundu), de l'épouse principale. Chez les Khasongé on peut épouser une veuve de l'autre clan mais pas une vierge. Le lien ainsi créé se montre le plus souvent supérieur à la parenté véritable et les sanctions surnaturelles qui appuyaient le pacte plus sévères que pour les liens de parenté. Un des cas les plus frappants est celui des Ganda étudié par Roscoe : un meurtrier peut trouver refuge auprès de son frère de sang même si sa victime est un parent de celui-ci.

[25] L'enquête de Beidelman (1963) sur les contrats de sang (quasiment disparus) auprès de vieux Kaguru du Tanganika lui permet néanmoins d'apporter de nouveaux arguments à l'analyse critique qu'avait faite Evans-Pritchard de l'expression 'fraternité de sang'. Le cas des Kaguru est particulièrement intéressant car ils sont matrilinéaires et l'appartenance au clan, contrairement à ce qui se passe avec les Zandé, est associée à la possession d'un sang commun (transmis par la mère). Or on ne considère pas un camarade de sang comme un parent matrilinéaire. La quantité de sang échangée, dit-on, est beaucoup trop faible.

[26] Si les Kaguru parlent malgré tout de parenté c'est, comme le dit Evans-Pritchard pour les Zandé, qu'ils n'ont pas d'autres façon possible de parler d'une relation qui comme la parenté est personnelle, forte et indissoluble même après la mort. Dans leur esprit il n'y a pourtant aucune confusion possible. La relation entre frères de sang est une relation entre égaux, supposée sans conflits d'intérêts tandis que les relations entre parents sont traversées par des rapports hiérarchique (aînés et cadets) et de concurrence pour le pouvoir et l'autorité. L'un peut même maudire l'autre et le

4 Selon Tegnaeus c'est le cas en Rhodésie dans de nombreuses tribus comme les Lemba. Dans certaines tribus comme les Lovedu, il s'agit seulement de certains mariages par exemple quand on 'craint les conséquences de l'union de certains sangs où quand un homme hérite de la femme de son frère mort' (1954 : 123) pour unir le couple et écarter le danger des relations sexuelles, car on dit qu'autrement l'homme peut attraper une maladie mortelle. Dans de nombreuses tribus comme les Tumbwe, le mariage devient indissoluble du fait de cet échange de sang. Chez les Ekoi c'est une façon de s'assurer la fidélité de la femme. Si elle est infidèle, le sang de son mari se révolte dans ses veines et provoque sa mort. Le redoublement du lien matrimonial (comme parfois ailleurs celui du lien de parenté consanguine) par un échange de sang montre bien que ce dernier n'est pas créateur de consanguinité. Sinon, comme le remarque Kuper (cité par Tegnaeus 1954 : 128) à propos des Swazi, les relations entre conjoints équivaudraient à un inceste. 
Citation: Vernier, Bernard (2006) 'Du bon usage de la parenté construite avec des humeurs corporelles (sang et lait) et quelques autres moyens', European Journal of Turkish Studies, Thematic Issue $N^{\circ} 4$, The social practices of kinship. A comparative perspective, URL : http://www.ejts.org/document623.html

To quote a passage, use paragraph (§)

réduire à l'état d'esclave. Ils peuvent se disputer à propos de leur participation aux compensations matrimoniales ou de leur droit de propriété. Le partenaire est, lui, quelqu'un qu'on ne roulera pas, à qui on ne refusera pas son aide (quand il la demande), qu'on ne volera pas et dont on ne prendra pas la femme.

[27] Si la relation de sang ressemble à une relation de parenté, c'est plutôt à celle qui existe entre générations alternées. Dans les deux cas, il existe une égalité et une intimité qui permet même de parler sexualité (un sujet souvent tabou avec d'autres parents). II est vrai que l'assimilation des 'camarades' est poussée au point que l'un traite la mère de l'autre comme sa propre mère. Mais la différence avec la parenté consanguine est si radicale qu'on peut épouser, comme chez les Zandé, une femme du clan de son partenaire.

[28] Sous certains rapports, on peut, comme Hoccart, comparer cette relation avec l'alliance matrimoniale: il s'agit d'établir des relations durables entre groupes. Mais, à la différence de la fraternité de sang, l'alliance matrimoniale est souvent traversée par des disputes à propos de la dot et du contrôle des enfants. Comme le disent certains : 'le mariage est une bataille'.

[29] Enfin, le rapprochement que fait Evans-Pritchard avec la relation à plaisanterie ne peut s'appliquer aux Kaguru. Les frères de sang n'ont pas de rapports à plaisanterie. Ces derniers lient soit des partenaires qui se rendent des services rituels et entretiennent des rapports très agressifs soit des parents (cousins croisés) entre qui une certaine agressivité est encouragée. Les frères de sang ne sont pas agressifs et ne se rendent aucun service rituel.

[30] Les relations entre groupes liés par un pacte de sang ne sont donc assimilables à aucune autre forme de parenté. II s'agit, pour des raisons commerciales ou politiques, de relier des non parents (personnes et groupes) par des liens étroits renforcés par des sanctions magiques automatiques qui sont un effet physiologique de la présence du sang de l'un dans le corps de l'autre.

[31] Le deuxième article de Denise Paulme qui date de 1968 a lui l'intérêt de mener une comparaison avec une autre institution existante en Afrique, celle de la classe d'âge. La relation entre co-initiés comporte, comme le pacte de sang, des devoirs d'entraide et de solidarité dans le cadre d'une réciprocité directe (non asymétrique comme souvent dans la famille où mon gendre me rendra les services que j'offre aujourd'hui à mon beau-père) et sur une base d'une parfaite égalité ('nous ne faisons plus qu'un'). Pacte de sang et classe d'âge ont en commun d'élargir le réseau de 
Citation: Vernier, Bernard (2006) 'Du bon usage de la parenté construite avec des humeurs corporelles (sang et lait) et quelques autres moyens', European Journal of Turkish Studies, Thematic Issue $N^{\circ} 4$, The social practices of kinship. A comparative perspective, URL : http://www.ejts.org/document623.html

To quote a passage, use paragraph $(\S)$

relation, de permettre une certaine indépendance par rapport aux parents, aux affins et aux aînés et de servir de support à une solidarité qui, à l'occasion, peut s'exercer contre la famille ou protéger Ego des entreprises hostiles de parents. Les co-initiés aident (de façon différente que les parents) le jeune à se marier. Ils fournissent les partenaires privilégiés pour toute entreprise commerciale. Certes le pacte est un contrat tandis que la classe est une institution, mais le jeune choisit parmi ses co-initiés des partenaires avec qui il aura des rapports très proches de ceux qu'il aurait eus avec un frère de sang. Pas besoin ici d'un échange de sang, ils sont déjà 'jumeaux'.

\section{Sur quelques pactes de paix nigérians}

[32] On sait par Meek (1925) que les pactes de paix étaient très courants au Nigéria entre individus et entre groupes. Les fils des chefs Zumper et Takum par exemple avaient le poignet incisé. Le sang qui coulait était recueilli dans une calebasse et mélangé avec de l'eau et les jeunes gens buvaient le mélange. Les chefs disaient alors : 'Notre sang est maintenant mélangé ; si dorénavant nous tuons ou vendons les membres de nos tribus respectives, que notre corps enfle et que nous en mourrions'. Chez les Ekoi (selon Talbot cité par Tegnaeus), on se sert du pacte de sang pour établir la paix ente les tribus mais aussi pour se protéger de la sorcellerie. Le parjure (qui s'efforce de nuire à quelqu'un) voyait le juju (pouvoir magique) du sang s'emparer de lui. Un cas cité est particulièrement intéressant. II s'agit d'un pacte de sang contracté entre tous les villageois pour que chacun soit désormais à l'abri de la sorcellerie des autres. Beaucoup de villageois mouraient. Le chef décida que tous les habitants devaient exécuter la cérémonie du sang. Au cours d'un rassemblement, on a incisé le corps de chaque villageois (y compris les bébés nés le jour même), recueilli à chaque fois quelques gouttes de leur sang dans une calebasse à demi pleine de grains de maïs. Puis le maïs fut partagé entre tous et mangé. Chez les Tiv (Bohanan cité par Tegnaeus), on contracte des pactes entre clans Tiv mais aussi entre clans Tiv et clans d'autres tribus. Ici le pacte semble avoir été conclu pour faciliter le mariage entre clans ou, pour le dire autrement, pour pouvoir s'inter-marier sans provoquer de frictions.

[33] Mon travail personnel a porté sur les environs de la ville de Jos située dans la partie centrale du Nigéria (Etat de Plateau). De nombreux groupes y sont encore liés par un pacte de paix qui comporte le plus souvent des interdits sexuels et matrimoniaux. L'accord est assez souvent fondé 
Citation: Vernier, Bernard (2006) 'Du bon usage de la parenté construite avec des humeurs corporelles (sang et lait) et quelques autres moyens', European Journal of Turkish Studies, Thematic Issue $N^{\circ} 4$, The social practices of kinship. A comparative perspective, URL : http://www.ejts.org/document623.html

To quote a passage, use paragraph (§)

sur un pacte de sang. Dans certains cas, on apporte une calebasse remplie d'eau. On fait une petite coupure au doigt des représentants des deux groupes et chacun plonge ce doigt dans l'eau. Les sangs se mélangent et les deux personnes boivent le liquide du récipient. Dans d'autres cas, ces représentants se coupent un peu l'avant-bras et se sucent mutuellement un peu de sang. Mais on peut aussi se contenter de boire une même eau dans laquelle a été plongée une herbe spéciale. Il en résulte une consubstantialité de type purement symbolique ou métaphorique entre les partenaires de l'accord.

[34] Ces prohibitions peuvent lier deux 'tribus', prises globalement, (ex. : les Boudji et les Anaguta) ou un sous-groupe d'une tribu (ex. : les Afiza de la tribu Afizéré) et l'ensemble des groupes appartenant à une autre tribu (ex. : ici, les Anaguta). Avec souvent des exceptions. L'interdit entre Afiza et Anaguta par exemple permet aux premiers d'épouser le sous-groupe Anagiran dont on dit qu'ils ne sont pas de vrais Anaguta parce qu'il s'est constitué à partir de familles provenant des groupes Dougeza et Ribina. De même l'interdit entre les Boudji et les Anaguta permet aux Boudji d'épouser les Anagiran et les Adougezi qui ne sont pas des Anaguta d'origine.

[35] Ces prohibitions peuvent aussi exister (comme chez les Boudji, les Anaguta, les Jere, les Taria, les Amo, etc.) entre certains groupes à l'intérieur d'une même tribu. Elles lient souvent certains sous-groupes dits d'origine et d'autres venus de l'extérieur. On aura une idée de la complexité des rapports entre les groupes locaux, dont la composition a été travaillée par les guerres, les famines et les migrations, si l'on sait, d'une part, que dans une population donnée (par exemple les Boudji), les groupes d'origine peuvent entretenir des relations de ce type avec certains des sous-groupe d'origine étrangère (comme dans ce cas les Agana), mais pas avec les autres et que les différents sous-groupes d'une même population, quand ils entretiennent ce type de relation, ne l'ont pas obligatoirement avec les mêmes tribus externes ou avec les mêmes sous-groupe de ces tribus.

[36] Ces interdits de relations sexuelles et matrimoniales ont été édictés, dit-on, entre groupes qui auparavant s'inter-mariaient, et qui, au moment de la prise de décision, étaient proches aussi bien géographiquement que culturellement (type de danse, scarifications, langue, culte etc.). Ils seraient en tout cas inconcevables entre des groupes aux traditions trop différentes. 
Citation: Vernier, Bernard (2006) 'Du bon usage de la parenté construite avec des humeurs corporelles (sang et lait) et quelques autres moyens', European Journal of Turkish Studies, Thematic Issue $N^{\circ} 4$, The social practices of kinship. A comparative perspective, URL : http://www.ejts.org/document623.html

To quote a passage, use paragraph (§)

[37] Les Agana qui sont actuellement un sous-groupe des Boudji viennent en réalité de la tribu Kurama. II y a très longtemps, fuyant la guerre, ils se sont réfugiés chez les Boudji : 'Nous voulons vivre avec vous'. Le chef Boudji pris de sympathie leur a répondu : 'c'est d'accord vous serez comme nos frères et donc vous ne pourrez plus prendre une femme chez nous ni nous chez vous. De sa main gauche il a pris de l'eau dans une calebasse avec une cuillère et l'a versée dans la bouche du vieux agenouillé devant lui qui représentait les Agana. A son tour, il s'est agenouillé et le vieil Agana lui a donné à boire, avec sa main gauche, l'eau de sa propre calebasse. C'est parce qu'ils ont utilisé la main gauche que toute transgression de l'interdit entraine automatiquement une maladie mortelle Ouparé. C'est la main qui est utilisée pour obtenir des choses néfastes. Elle est du côté de ce qui est diabolique [evil]. On ne donne jamais la main gauche pour dire bonjour'.

[38] Les Anaguta entretiennent une relation de ce type ${ }^{5}$ appelée Apari avec les Afiza, sousgroupe de la 'tribu' Afizéré. Les deux sociétés sont patrilinéaires. Selon un Anaguta, tout a commencé ainsi :

[39] 'Quand les Afiza migrèrent du village Laminga vers Jos ils ont vu une fumée qui s'élevait au-dessus d'une montagne (...) et ils ont entendu le bruit d'une femme qui pilait. En se dirigeant de ce côté, ils ont découvert les Anaguta qui s'étaient établis là avant eux alors qu'ils croyaient être les premiers arrivants. Les Afizeré dirent alors aux Anaguta : 'Soyons frères et vivons en paix!' et ils décidèrent de vivre ensemble. Plus tard une guerre éclata entre les Anaguta et les Rukuba. Ces derniers tuèrent un grand nombre d'Anaguta. Les Afizeré se sont dits 'les Rukuba ont tué presque tous nos frères. II faut sauver ceux qui restent'. Ils ont caché les Anaguta dans une vallée, leur ont offert leurs propres scarifications et leur ont appris leurs danses. Quand les Rukuba sont arrivés en poursuivant les Anaguta, ils ont vu des gens avec des marques Afizere et les ont laissés en paix, persuadés qu'ils avaient tué tous les Anaguta. Plus tard, les aînés des deux groupes se sont rencontrés et ils ont décidé ceci: 'Nous ne devons plus jamais nous combattre et nous faire la guerre. Qu'aucun d'entre nous n'ait même le droit de voir le cadavre d'un membre de l'autre groupe!'. Les Anaguta ont déclaré : 'Nous sommes vivants grâce à vous. Maintenons de solides liens entre nous. Pour cela il ne doit plus y avoir ni mariage ni relation sexuelle entre nous. Les Afizeré ont

5 On nomme l'autre mon Oupari (au singulier) mes Apari (au pluriel) chez les Anaguta / Aparé chez les Budji, Ripari chez les Afizéré, Itari en Haoussa prononcé souvent Itaré chez les Jere. L'utilisation fréquente du mot Itari par les autres groupes montre la domination du Haoussa dans toute cette région du Nigéria. Rappelons que chez les Dogons l'alliance et l'allié porte le même nom : mangou. 
Citation: Vernier, Bernard (2006) 'Du bon usage de la parenté construite avec des humeurs corporelles (sang et lait) et quelques autres moyens', European Journal of Turkish Studies, Thematic Issue $\mathrm{N}^{\circ} 4$, The social practices of kinship. A comparative perspective, URL : http://www.ejts.org/document623.html

To quote a passage, use paragraph (§)

répondu : 'Puisque nous sommes frères, faisons la paix pour toujours! Interdisons les mariages et les relations sexuelles entre nos deux groupes car les femmes sont une importante source de conflit'.

[40] Les récits se ressemblent tous et ressemblent fort à tous ceux recueillis par les ethnologues depuis le début du XXème siècle. Un groupe extérieur abandonne son emplacement habituel à cause de la guerre, d'une épidémie ou de la mort rapprochée de nombreux enfants. II se réfugie chez ou près d'un autre groupe avec lequel il établit une relation Apari. Chez les Anaguta, les sous-groupes d'origine extérieure qui se sont réfugiés chez eux sont devenus Anaguta mais s'ils peuvent se marier avec les autres groupes Anaguta qui, comme eux, viennent de l'extérieur, ils ne le peuvent pas avec les sous-groupes qui sont Anaguta depuis l'origine et avec lequel ils entretiennent une relation Apari. Parfois un sous-groupe extérieur n'a de relation Apari qu'avec le sous-groupe qui l'a accueilli. Souvent ce sont les débiteurs qui proposent le pacte : 'car vous les avez sauvés, vous vous êtes occupés d'eux et maintenant ils vous prennent pour un père ou un dieu et ils promettent de ne rien prendre avant que vous ne le donniez et de ne jamais se marier avec vous car ils disent que nous sommes un'.

[41] Dans certains cas, la relation implique l'interdiction du mariage avec les femmes de l'autre groupe (interdiction du vol des femmes) mais pas avec ses filles. C'est le type de relation établie entre les Boudji et un sous-groupe Afizéré, les Amabour, appelé aussi par les premiers les Gouasch d'après leur village d'origine. Sur l'origine de cette relation, on raconte ceci :

[42] 'Une femme Boudji avait décidé de quitter son mari. Elle est allée à Gouasch où vivaient les Amabour. Elle était très belle et séduisante. Un Amabour lui a demandé d'où elle venait. Elle s'est contentée de répondre 'de très loin'. II lui a proposé de l'héberger et elle l'a suivi. II lui a donné un endroit où dormir, l'a nourrie et l'a gardée chez lui. Le mari Boudji la cherchait et il demandait partout si on avait vu sa femme. Quelqu'un lui répondit qu'il avait vu une femme se diriger vers l'endroit où vivaient les Amabour. II a pris cette direction et en route il a rencontré un homme Afiza. II lui a demandé : 'avez vous vu ma femme?'. L'autre lui a répondu : 'une femme est venue vers moi et je l'ai hébergée. Peut-être estce la vôtre?'. L'homme Boudji suivit l'Afiza chez lui et il y découvrit sa femme. II lui demanda alors en montrant l'Afiza 'cet homme t'a-t-il seulement hébergée ou t'a-t-il prise pour femme?' La femme lui a répondu: 'Il m'a hébergée et protégée. Mais nous n'avons pas eu de relation sexuelle de tout ce temps'. Le Boudji dit alors à l'Amabour: 'A partir de maintenant faisons une promesse. Aucun Boudji ne pourra prendre la femme d'un Afiza et aucun Afiza la femme d'un Boudji. Si 
Citation: Vernier, Bernard (2006) 'Du bon usage de la parenté construite avec des humeurs corporelles (sang et lait) et quelques autres moyens', European Journal of Turkish Studies, Thematic Issue $N^{\circ} 4$, The social practices of kinship. A comparative perspective, URL : http://www.ejts.org/document623.html

To quote a passage, use paragraph $(\S)$

quelqu'un transgresse la loi, il en supportera les conséquences ${ }^{6}$. Ils ont cependant décidé qu'ils pourraient chacun épouser les filles de l'autre groupe'.

[43] Quand on demande pour quelles raisons on établit une relation Apari, la réponse stéréotypée est 'pour qu'il n'y ait pas de conflit'. Et quand on demande de façon plus précise pourquoi on interdit les relations sexuelles et matrimoniales on obtient des réponses qui se ressemblent :

- 'c'est pour rompre avec l'ancienne coutume qui était de se voler mutuellement des femmes ${ }^{7}$. Ces vols étaient la cause de beaucoup de guerre'.

- 'c'est pour qu'il n'y ait pas de conflit à propos des femmes'.

[44] La prohibition des relations sexuelles et du vol de femmes entre les deux groupes implique aussi qu'il est interdit par exemple à un Afiza et un Anaguta d'avoir des relations simultanées avec une même femme quand bien même cette femme appartiendrait à un troisième groupe qui n'est Apari avec aucun des deux. Si cela arrive et que l'on s'en aperçoit, l'un des hommes doit se désister pour que l'autre puisse continuer la relation. En supprimant les situations de rivalité sexuelle on supprime ici encore les occasions de conflit. II est interdit aussi de prendre une femme d'un troisième groupe qui a été mariée à un Apari même si elle en est divorcée. Si le prétendant tient vraiment à cette femme, il devra attendre pour la prendre qu'elle se remarie dans un autre groupe, qu'elle y reste au moins un an (on considère qu'au but de cette période elle n'a plus rien de commun avec l'Afiza. Elle est devenue 'clean') et divorce à nouveau. Ces interdits dont on trouve des équivalents dans toute la région ont pour fonction d'éviter des occasions de rivalité et de conflits sexuels et matrimoniaux entre Apari.

[45] Si l'on peut affirmer avec force que ces interdits sexuels ont bien pour objectif, comme le disent les Nigérians, d'assurer la paix, c'est qu'ils font partie d'un ensemble de prohibitions qui toutes assument explicitement cette même fonction : interdiction de se faire la guerre, de se tuer (on dit que ce n'est jamais arrivé volontairement), de se battre (on dit que c'est impensable), de se mettre en

6 La mort à la suite d'une maladie qui est rendue visible par un gonflement du ventre.

7 Chez les Anaguta, on nomme Randal l'action de voler une femme. Cette pratique existe encore entre Anaguta. Le mari volé se contente de battre le voleur (jamais sur la tête) s'il le trouve, mais souvent celui ci est prévenu et se sauve à temps. Il arrive parfois qu'il saccage sa maison. 
Citation: Vernier, Bernard (2006) 'Du bon usage de la parenté construite avec des humeurs corporelles (sang et lait) et quelques autres moyens', European Journal of Turkish Studies, Thematic Issue $N^{\circ} 4$, The social practices of kinship. A comparative perspective, URL : http://www.ejts.org/document623.html

To quote a passage, use paragraph $(\S)$

colère ou de se disputer verbalement, de se voler, de se maudire ou d'utiliser la sorcellerie les uns contre les autres. L'interdit de se faire la guerre s'étend au fait de contempler le cadavre d'un Apari.

[46] C'est aussi pour supprimer les occasions de querelle que l'on s'attache à maintenir une stricte égalité entre les partenaires dans différentes situations de la vie quotidienne : 'Si un Afizeré donne quelque chose avec la main droite, l'Anaguta doit le prendre de la main droite. S'il la donne de la main gauche, il l'a prendra de la main gauche. II n'y aura dans ce cas aucune insulte entre eux. Si l'Anaguta tend la main droite pour saluer, l'Afizeré donne aussi la main droite et la gauche si c'est la gauche car la relation doit être égale'.

[47] Les Afiza et les Anaguta, comme beaucoup de groupes en relation Apari, entretiennent une relation d'amitié spéciale et s'invitent aux mariages, aux enterrements et de façon générale aux fêtes. On les considère comme des parents (Atemeu en Eguta). Dans une fête organisée dans un des deux groupes (comme la cérémonie de la moisson) : 'les Apari qui sont là et dansent peuvent se déshabiller complètement tant ils sont joyeux d'être là. Ils ont établi entre eux une relation répandue dans le monde entier et que les ethnologues ont coutume de nommer 'parenté à plaisanterie'. A partir d'une étude comparative Radcliffe-Brown donne à cette relation la fonction d'établir les conditions d'une coexistence harmonieuse des groupes ou plus exactement celle, pour reprendre ses termes, de maintenir la conjonction dans un contexte de disjonction possible. C'est précisément la fonction qu'elle remplit ici. II est bien considéré de s'insulter: 'On peut se moquer les uns des autres, se bousculer, se battre, se fâcher, et se moquer mais à condition que ce soit pour rire'. Cette relation à plaisanterie permet de s'emparer de ce qui plaît (volaille, caisse de bière etc.) chez un Apari. L'ancienne coutume du vol réel de femme est remplacée par un vol rituel : 'On s'amuse à prendre la femme d'un Apari par la main et on l'emmène sur plusieurs mètres. Le mari rit de bon cœur, car il sait que je ne ferai rien de mal avec elle'.

\section{Des parents souvent plus utiles que les parents par alliance}

[48] La relation de parenté Apari comporte comme toute relation de parenté une obligation d'entraide multiforme. Sous ce rapport elle est, dit-on souvent, plus pratique, libre et importante que la relation d'alliance : 
Citation: Vernier, Bernard (2006) 'Du bon usage de la parenté construite avec des humeurs corporelles (sang et lait) et quelques autres moyens', European Journal of Turkish Studies, Thematic Issue $\mathrm{N}^{\circ} 4$, The social practices of kinship. A comparative perspective, URL : http://www.ejts.org/document623.html

To quote a passage, use paragraph $(\S)$

- 'Avec les Apari, je me sens libre je n'ai aucune timidité. Je me sens plus libre qu'avec un beau-frère. Devant la famille de ma femme j'ai honte. Surtout avec ma belle-mère, il n'y a pas de liberté. Au début on ne se parle même pas surtout avec la belle-mère jusqu'au moment où le silence est rompu par un don de tabac local'.

- 'J'ai une relation à plaisanterie (joking relation) avec les cadets et cadettes de ma femme mais j'ai honte devant ses aînés. Si on a échangé des sœurs on est des égaux mais sinon les alliés sont mes supérieurs car ils m'ont donné ma femme'.

- 'Les Apari, même s'il s'agit du roi, je peux aller sans problème manger chez eux. Les alliés ne nous aident que si on invite un groupe de travail que l'on nourrit. Mais on peut faire appel aux Apari à n'importe quel moment. Et puis avec les alliés c'est la famille du mari qui va travailler chez la famille de la femme, pas l'inverse'.

- 'Si j'ai besoin de quelqu'un pour un travail, je choisirai si je peux un Apari. Avec lui je n'aurai pas de problème'.

- 'On a honte de demander de l'aide à la famille de sa femme. Les Apari on peut faire appel à eux librement'.

- 'Si on a besoin de quelque chose (chèvre, médecine) c'est mieux de s'adresser à des Apari. Ils ne peuvent pas vous rouler et vous faire payer une médecine inefficace, car ils considèrent que cette relation est sacrée'.

- 'Un allié peut toujours me ressortir un jour que je lui ai demandé de l'aide. Un Apari, non. Un Apari tient mes secrets, par exemple si je lui demande une médecine pour une maladie sexuelle, même si c'est une femme. II ne m'expose pas alors qu'un parent peut le faire. Un beau-frère peutêtre pas mais une belle-sœur sûrement'.

- 'Si je veux une fille, j'utilise un Apari comme messager. A la différence d'un parent, il ne pourra pas prendre la fille pour lui'.

[49] Ajoutons que cette entraide entre Apari peut avoir une expression politique. Les Anaguta et les Afizeré sont encore liés actuellement par une alliance politique. Selon un principe d'alternance, le chef administratif (chairman) du district nord de Jos est tantôt Anaguta tantôt Afizeré. 
Citation: Vernier, Bernard (2006) 'Du bon usage de la parenté construite avec des humeurs corporelles (sang et lait) et quelques autres moyens', European Journal of Turkish Studies, Thematic Issue $N^{\circ} 4$, The social practices of kinship. A comparative perspective, URL : http://www.ejts.org/document623.html

To quote a passage, use paragraph $(\S)$

[50] Mais l'importance fonctionnelle de la relation Apari vient aussi de ce qu'elle ne se contente pas d'établir les conditions de la paix entre deux groupes, elle est aussi utilisée pour maintenir la concorde au sein de chacun des deux groupes. L'Apari est un médiateur d'une efficacité exceptionnelle. Quand deux personnes d'un même groupe se battent si un Apari survient et leur dit de s'arrêter ils sont, pour ainsi dire, condamnés à lui obéir. L'Apari leur fait honte : 'vous êtes frère et sœur, cessez de vous battre!' et il plaisante 'pourquoi tu te bats avec ta sœur?'. Dans un conflit qui porte sur des droits de propriétés entre deux membres du même groupe (par exemple Afiza) l'Apari (ici Anaguta) qui connaît le vrai propriétaire est capable d'obliger l'usurpateur à céder d'une simple injonction.

[51] Dans cette fonction de maintien de la paix, la relation Apari est dite, de l'avis de tous, bien supérieure aux relations de parenté même consanguines et ceci dans trois domaines ${ }^{8}$ :

[52] 1- Les disputes entre conjoints. Un Anaguta explique : 'Si on se dispute avec sa femme celle-ci peut aller, en toute sécurité, chez un ami Apari. II n'a pas le droit d'avoir des rapports sexuels avec elle. Plus tard il la ramènera chez son mari et offrira à celui-ci de la nourriture pour le remercier parce que cette histoire a renforcé leur relation. II demandera alors au coupable de ne plus créer de dispute'. Mais pourquoi la femme préfère aller chez un Apari plutôt que de retourner chez son père? Elle sait que 'si elle va chez son père elle aura une deuxième querelle avec lui. L'Apari, lui, n'a pas le droit de se disputer avec elle. En plus elle est tout à fait en sécurité chez lui car son père ne peut pas venir la récupérer de force chez un Apari. Enfin, elle est sûre que quand l'Apari la reconduira chez son mari, l'affaire se calmera car le père et le mari sont obligés d'écouter et de suivre les recommandations d'un Apari. Ils ne peuvent aller contre sa volonté d'apaisement. Un mari n'écoute pas ce que disent ses beaux-parents, mais il écoute ce que dit un Apari'.

[53] 2- Les attaques de sorcellerie. Les attaques les plus dangereuses proviennent des parents (père et mère) ou d'un membre du groupe d'appartenance patrilinéaire d'Ego par exemple germain ou demi-germain surtout de même père, oncle, tante, grand parent, enfant et petit enfant etc. Un enfant, par exemple, peut en vouloir à son père qui ne lui donne pas ses richesses. La tante paternelle peut 'manger' Ego. On est aussi menacé par certains parents par alliance. Une femme peut 'manger' son mari (car 'ils ne font qu'un'), sa co-épouse (les deux femmes 'appartiennent à une

8 Ceci est vrai également pour les relations Apari entre sous-groupes d'une tribu comme les Taria, les Kagoro, les Amo, les Boudji, etc. 
Citation: Vernier, Bernard (2006) 'Du bon usage de la parenté construite avec des humeurs corporelles (sang et lait) et quelques autres moyens', European Journal of Turkish Studies, Thematic Issue $N^{\circ} 4$, The social practices of kinship. A comparative perspective, URL : http://www.ejts.org/document623.html

To quote a passage, use paragraph (§)

même famille') ou les enfants de celle-ci ('ils sont comme ses propres enfants'). Un enfant doit aussi craindre ses parents maternels (oncles, tantes et grands-parents) car ceux-ci s'ils veulent toucher la mère peuvent décider de l'atteindre dans ce qu'elle possède : son enfant. La mère et l'enfant, dit-on, ne font qu'un. II semble cependant que, dans certains groupes, l'oncle maternel puisse protéger d'une certaine façon de la sorcellerie. Si je suis attaqué en sorcellerie par ma famille paternelle, je me réfugie chez lui car on sait que la sorcellerie ne peut entrer chez lui sinon il reprendra sa sœur et ses neveux et nièces ainsi que la compensation matrimoniale qui a été donnée. On sait qu'il a ce pouvoir. La grand-mère maternelle peut manger les enfants de sa fille si elle déteste sa fille. Les frères et sœurs de la grand-mère maternelle ne peuvent pas, eux, atteindre l'enfant. S'ils détestent leur sœur, ils peuvent 'manger' la mère d'Ego, mais pas Ego lui-même. La famille du mari peut atteindre la femme mariée car celle-ci appartient maintenant au groupe du mari, mais ils ne peuvent pas atteindre les frères et sœurs de cette femme. La famille de la femme ne peut, elle, atteindre le mari et sa famille car le mari n'appartient pas à leur groupe. Enfin une femme peut atteindre par sorcellerie une autre femme avec qui elle n'a aucune parenté, mais qui a des relations sexuelles avec le même homme : 'elles sont à lui toutes les deux'. Et réciproquement un homme peut atteindre un homme non parent qui a des relations avec la même femme que lui et dont, pour cela, il souhaite la mort. De façon générale, quand on est 'mangé' par un étranger c'est que celui-ci a un complice dans la famille. Par exemple, un membre de la famille maternelle peut s'entendre avec l'oncle paternel de l'enfant pour 'l'attraper'.

[54] Seule la maison d'un Apari fournit un vrai refuge. Si quelqu'un s'efforce d'atteindre un parent réfugié chez un Apari il mourra, car 'il n'a pas le droit de faire entrer la sorcellerie dans cette maison'. II arrive que l'Apari donne une médecine à boire mais la relation Apari protège essentiellement par elle-même et d'une protection durable car l'Apari raccompagne toujours son ou sa protégée dans sa famille en disant: 'Cette personne m'appartient encore, même si je vous la ramène. Si quelqu'un d'entre vous la détruit, vous allez tous mourir'.Cette menace suffit pour faire cesser les attaques.

[55] On donne dans tous les groupes beaucoup d'importance à l'interdit de sorcellerie. L'une des personnes interrogées a même prétendu que c'est en grande partie pour se défendre de la sorcellerie qu'on a créé des relations Apari : 'C'est à cause de la méchanceté des gens'. II était clairement sous-entendu qu'elle utilisait la sorcellerie. 
Citation: Vernier, Bernard (2006) 'Du bon usage de la parenté construite avec des humeurs corporelles (sang et lait) et quelques autres moyens', European Journal of Turkish Studies, Thematic Issue $N^{\circ} 4$, The social practices of kinship. A comparative perspective, URL : http://www.ejts.org/document623.html

To quote a passage, use paragraph $(\S)$

[56] 3- Les guerres. On peut se réfugier chez un Apari en cas de menace de toute sorte, même provenant de sa propre tribu. C'est ce que me dit un Anaguta : 'je suis un Anagomo du village Andoro et je peux me réfugier dans tous les villages où j'ai une relation Apari, je serai protégé et personne ne pourra me toucher. II y avait avant beaucoup de guerre et les gens avaient besoin de groupes où se réfugier'.

[57] Les interdits qui régulent les relations entre Apari puisent une grande partie de leur efficacité dissuasive dans le fait que toute transgression (voler ou au contraire refuser de se laisser 'voler' dans le cadre de la relation à plaisanterie, se battre, se disputer, utiliser la sorcellerie, avoir des rapports sexuels, etc.) provoque par elle-même la mort du coupable. Si, comme on dit, 'Itari nous attrape', on est condamné à mourir et la menace s'étend aux autres membres de la famille. Les victimes d'Itari sont atteintes de saignements du nez et des organes sexuelles. Leurs jambes et leurs bras maigrissent, leurs ventres gonflent progressivement au point qu'ils ne peuvent plus respirer ('comme s'ils étaient enceints' a précisé quelqu'un) jusqu'à ce qu'une mort horrible s'en suive. Les 'animistes', et même une grande partie des personnes christianisées interrogées, considèrent qu'il est beaucoup plus grave (au sens où cela les effraie beaucoup plus) de coucher avec une femme Apari que de coucher avec une parente (par alliance ou même consanguine) interdite. C'est vrai dans tous les groupes où nous avons fait des entretiens (Anaguta, Jere, Boudji, Taria, etc.).

[58] L'inceste est bien stigmatisé, mais ses conséquences sont moins graves que la transgression des normes qui régissent les rapports entre Apari. Quelqu'un qui couche avec sa sœur de père et de mère et qui est surpris est détruit socialement: sa parole ne vaut plus rien dans les assemblées. Dans certains groupes de la région, il est déshérité voir chassé ou bien il s'exile de luimême avant qu'on ne le chasse. Mais il ne risque ni de mourir ni même de tomber malade. Son acte, si honteux soit-il, ne donne lieu souvent, comme chez les Anaguta et les Boudji, à aucun rite de purification. Si avoir des relations sexuelles avec une femme Apari est plus grave qu'avec un parent patrilinéaire c'est parce que selon la belle expression d'un Boudji 'coucher avec un Agana (groupe Apari pour les Boudji), c'est comme si l'on invitait la mort chez soi' la mort à la suite d'une maladie qui est rendue visible par un gonflement du ventre9.

9 Pour une description plus poussée de ces pactes de paix, du rituel de purification en cas de transgression et pour une analyse de l'importance d'une prise en considération de ces pactes pour mieux comprendre la prohibition de l'inceste ordinaire, on peut se reporter à mon article (Vernier 2005a). 

quelques autres moyens', European Journal of Turkish Studies, Thematic Issue $\mathrm{N}^{\circ} 4$, The social practices of kinship. A comparative perspective, URL : http://www.ejts.org/document623.html

To quote a passage, use paragraph (§)

\section{La parenté de lait}

[59] L'alliance par le lait semble avoir été également extrêmement répandue. Comme le rappelle Jane Khatib-Chahidi (1992), à qui nous empruntons les renseignements qui suivent, chez les Masaï de l'est africain, quand deux tribus ennemies voulaient établir une paix durable chacune amenait une vache avec un veau et une femme avec un bébé. Les deux vaches étaient échangées et le bébé ennemi était allaité par la femme Masaï et inversement. On peut ajouter que l'échange de lait existait aussi en Afrique chez Zoulou et les Dagas. Les royal Rajputs (d'après Lyall 1882) choisissaient habituellement la nourrice de leurs enfants dans une tribu pastorale. La famille de la nourrice avait un statut héréditaire de parents par le lait dans le clan royal et les frères de lait de l'enfant jouissaient d'un grand pouvoir à la cour. Dans le Hindu Kush (Biddulph 1880) les familles gouvernantes des tribus récemment converties à l'islam et qui ont gardé beaucoup de coutumes non islamiques (comme l'égalité entre hommes et femmes devant l'héritage) plaçaient leur enfant chez une nurse qui l'élevait plusieurs années. En retour, le mari de la nurse et les hommes de la famille obtenaient des postes et étaient liés par un certain nombre d'obligations à l'égard de la famille gouvernante. Dans les clans Ashimadek de Chitral dans le Hindu Kush, chaque enfant était allaité à tour de rôle par chaque femme du clan en état de la faire. Cette circulation continuelle des enfants entre les mères renforçait l'unité tribale. En Géorgie on utilisait la parenté de lait pour avoir des partenaires de confiance dans le commerce avec des régions ennemies. Le Géorgien chrétien envoyait un nouveau-né à son partenaire musulman du nord Caucase au moment où la femme de celui-ci allaitait. Ce musulman utilisait le même procédé mais avec un autre partenaire. L'enfant restait dans la famille jusqu'à $10 / 11$ ans puis était renvoyé chez lui pour montrer qu'on ne voulait pas l'exploiter comme main-d'œuvre gratuite. II s'agissait d'un investissement à long terme : les germains de lait pouvaient parler la même langue et chacun connaissait les traditions de l'autre ce qui favorisait la coopération. Cette relation était durable et les descendants des deux partenaires ne pouvaient pas se marier pendant sept générations même si leur religion le leur permettait (Dragadze 1988). Les chrétiens slaves de l'est et du sud de l'Europe utilisaient la parenté de lait (avec d'autres parentés fictives) pour interdire les intermariages (Maine 1891, 1893 et Filipovic 1963 cité par Fortier 2001). Chez les Slaves du sud on l'utilisait parfois pour établir une parenté entre musulmans et chrétiens. Selon Corbier (1999) le code Theodosius interdit la mise en nourrice des enfants des villes 
Citation: Vernier, Bernard (2006) 'Du bon usage de la parenté construite avec des humeurs corporelles (sang et lait) et quelques autres moyens', European Journal of Turkish Studies, Thematic Issue $\mathrm{N}^{\circ} 4$, The social practices of kinship. A comparative perspective, URL : http://www.ejts.org/document623.html

To quote a passage, use paragraph $(\S)$

chez les bergers pour casser la complicité des citoyens avec les brigands et elle rappelle que la femme de Caton avait allaité des enfants d'esclaves pour que son fils puisse avoir des frères de lait loyaux.

\section{Marcy}

[60] William Robertson Smith avait montré (1885 et 1914) le caractère fictif de l'idée de solidarité de la tribu arabe fondée sur l'appartenance à un groupe agnatique issu d'un même ancêtre masculin en ligne masculine. Les tribus arabes sont travaillées par des phénomènes de fission et de fusion qui les remodèlent continuellement. Surtout, il existe toute une série de procédés d'intégration des étrangers à la tribu ou d'établissements d'alliances comme les pactes de sang, les pactes de fraternité et diverses formes de pactes de protection ou de clientélisme.

[61] Dans un article important sur les Berbères, Marcy (1936), dont nous suivrons le texte pas à pas, observe que s'ils ont 'une conception généalogique du groupe' (clan, tribu etc.) en réalité ' la filiation des membres individuellement considérés avec l'ancêtre est presque toujours fictive, au moins pour la plupart d'entre eux. Nul d'ailleurs ne s'y trompe ... L'éponymie n'est qu'une fiction sociale commode (Marcy 1936: 961)'. C'est que 'le groupe social s'accroît par bien d'autres procédés que celui de la génération naturelle : naturalisation d'étrangers établis sur le territoire de la tribu, soit au titre d'adjar, 'protégé', soit à celui d'amazzal c'est-à-dire de 'fils adoptif'; adoption par un chef de famille d'enfants mineurs; fractions entières artificiellement agrégées par le moyen des différents systèmes d'inféodation, dont la tâd'a, l'alliance par co-lactation, est le plus représentatif (Marcy 1936 : 960). Dans tous les cas, il s'agit 'de faire naître juridiquement (par droit divin autant que civil) par la manifestation d'un symbolisme approprié, les effets ordinairement attachés aux liens de la parenté naturelle (1936 : 961). La menace de châtiment céleste garantissait l'observance de ces pactes sociaux. Concernant la tâd'a, Marcy en parle comme d' 'une sorte d'alliance inter-tribale, à caractère magico-religieux très prononcé, qui - semble-t-il- se concluait essentiellement à l'origine, par le recours au procédé symbolique de la co-lactation' (Marcy 1936: 958). Cette institution était 'destinée - par imitation magique du phénomène de la parenté maternelle - à faire naître, entre tous les participants à l'alliance, des liens ethniques artificiels, susceptibles d'entraîner, des uns aux autres, les mêmes obligations juridiques et morales qui, normalement s'imposent aux 
Citation: Vernier, Bernard (2006) 'Du bon usage de la parenté construite avec des humeurs corporelles (sang et lait) et quelques autres moyens', European Journal of Turkish Studies, Thematic Issue $N^{\circ} 4$, The social practices of kinship. A comparative perspective, URL : http://www.ejts.org/document623.html

To quote a passage, use paragraph (§)

personnes unies par la communauté de sang' (Marcy 1936 : 960)10. Les partenaires liés par la tâd'a sont 'des groupements assez étendus, généralement deux fractions ou même deux tribus. Le formalisme primitif accompagnant la conclusion du contrat - et qui semble aujourd'hui tombé en désuétude - consistait à faire réciproquement échange, entre les deux groupes de contractants, de vases remplis de lait dont le contenu était absorbé par les assistants'. Chez les Aït Mt'ir qui, selon Marcy, semblent avoir conservé le rituel dans ses détails 'on choisit, parmi chacune des deux parties, sept femmes nourrissant un enfant, dont on prélève le lait dans un bol qui circule ensuite de mains en mains et où chaque individu présent trempe à tour de rôle ses lèvres' (1936 : 967). C'est dire que l'absorption de lait est purement symbolique et qu'il serait absurde d'en déduire une identité substantielle des deux tribus ou fractions de tribu. Si une telle identité est supposée, il s'agit d'une fiction sociale construite pour les besoins de la cause. Marcy mentionne aussi une utilisation du lait dans le cadre de l'adoption. II a trouvé dans le Bayân un témoignage de l'Antiquité qui se rapporte à la période de conquête du Maghreb par les envahisseurs arabes après la bataille de Meskiana, où l'émir arabe H'asan fut défait par les troupes berbères de la Kahena (en 698 de notre ère). La Kahena adopta un des prisonniers arabes du nom de Khaled : 'Tu es l'homme le plus beau, le plus brave, que j'ai jamais vu, lui dit-elle, selon le Bayân ; aussi je veux te donner de mon lait pour qu'ainsi tu deviennes le frère de mes deux fils ; chez nous tous, Berbères, la parenté de lait confère un droit réciproque d'hérédité'. En conséquence, elle prit de la farine d'orge qu'elle aggloméra avec de l'huile et qu'elle plaça sur ses seins ; puis appelant ses deux enfants, elle la leur fit manger avec Kahled sur sa poitrine et leur dit : 'Vous êtes devenus frères'.

[62] Dans d'autres tribus (Marcy 1936: 967), il existe encore maintenant une institution semblable, sous forme plus atténuée de liens individuels, entre membres de deux fractions par moyen de tirage au sort effectué avec une pièce de vêtement, sandale ou turban ou même de simple buchette de bois. Des Marabouts et Chorfa appartenant aux deux parties prononcent certaines paroles qui donnent une consécration religieuse au pacte. La cérémonie est suivie d'un rite de commensalité probablement moins accessoire que ne le laisse supposer Marcy : les chefs des tentes s'hébergent mutuellement et s'offrent 'à tour de rôle une sorte de repas communiel, analogue en tout point à celui qui consacrait l'alliance chez les anciens sémites'. Les parties en présence par une 
Citation: Vernier, Bernard (2006) 'Du bon usage de la parenté construite avec des humeurs corporelles (sang et lait) et quelques autres moyens', European Journal of Turkish Studies, Thematic Issue $N^{\circ} 4$, The social practices of kinship. A comparative perspective, URL : http://www.ejts.org/document623.html

To quote a passage, use paragraph (§)

sorte de fiction, très probablement perçue comme telle, sont censées avoir été nourries ensemble par une femme identique et se disent 'frères de tad'a' (1936 :968). Cette forme de pacte est appelée dans le Maroc central 'petite tâd'a' par opposition à l'ancienne tâd'a qui utilisait le symbolisme du lait. On l'appelle aussi 'taussart ce qui veut dire vieille femme, à bien comprendre, c'est l'ancêtre, l'aïeule, dont la personnalité fictive est à la souche du groupe social nouveau'11. II est interdit de se marier entre les deux parties (fraction de tribu, tribus ou familles) qui contractent ce pacte. Marcy observe que Tâd'a est personnifiée comme si elle était une divinité. On notera seulement qu'elle est censée intervenir personnellement pour sanctionner le parjure conjointement avec Allah dont on invoque toujours le nom.

[63] L'auteur consacre la fin de son article à énumérer les usages de ces pactes de colactation. Les obligations sont analogues, toute chose étant égale par ailleurs, à celle de la foi jurée chez nous au Moyen Age : obligation mutuelle d'assistance (hospitalité à l'égard de celui qui est de passage sur le territoire de la tribu), prohibition de tout pillage et de toute déprédation entre les deux groupes (Marcy 1936: 970)12, commerce équitable (interdiction de tout profit injustifié réalisé aux dépens de l'allié), gratuité du service rendu (le chef de tente chez qui une femme en fuite a cherché refuge, ne doit point faire payer au mari poursuivant l'indemnité de mekhsur exigible en pareil cas). Dans les rapports entre les partenaires, chacun est sûr d'obtenir de l'autre un comportement obéissant à la logique d'une 'stricte justice': 'le chef de tente se doit ainsi, d'honneur, de répondre sur ses biens propres de tout préjudice qui pourrait être causé par ses contribules à son u-tâd'a ; en tout état de cause il est tenu de s'entremettre à titre d'arbitre en vue d'arriver à un règlement à l'amiable'. Les garanties de droit commun, en cas de litiges, sont renforcées : des conventions augmentent la charge de la preuve et généralement le nombre des notables devant prêter serment. Enfin la personne de l'u-tâd'a est sacrée pour son frère d'alliance (Marcy 1936 : 971) et par extension sa famille, ses biens meubles ou immeubles: 'sa tente, ses terrains de pâturage, doivent être scrupuleusement respectés (...) l'alliance détermine, sur le terrain, autour de la tente de celui qui y participe, une zone d'interdit magique où son frère - protégé lui-même de façon identique par la toute

11 II n'est cependant pas totalement absurde de penser que cette appellation de 'vieille femme ' renvoie non à la mère nourricière ancestrale mais tout simplement à une femme sans lait puisque ce qui caractérise cette tâd'a c'est précisément l'absence d'utilisation du lait.

12 Dans le cas d'un pacte à caractère interindividuel et d'une guerre entre leurs tribus il faut tout faire pour épargner le partenaire et le faire épargner par les siens ce qui tend à montrer que la solidarité entre partenaires de lait tend à être parfois plus forte que la solidarité d'intérêts entre membres de la tribu. 
Citation: Vernier, Bernard (2006) 'Du bon usage de la parenté construite avec des humeurs corporelles (sang et lait) et quelques autres moyens', European Journal of Turkish Studies, Thematic Issue $N^{\circ} 4$, The social practices of kinship. A comparative perspective, URL : http://www.ejts.org/document623.html

To quote a passage, use paragraph (§)

puissance de la tâd'a - ne peut, sauf permission du propriétaire, absolument pas toucher sans s'exposer à de redoutables châtiments célestes. Cette zone englobe tout l'espace de terrain qui est nécessaire pour pourvoir aux besoins journaliers de la tente, soit en herbe pour les animaux, soit en bois de chauffage, soit même en buissons d'épines pour l'aménagement du parc à bétail et la protection des meules de céréales. II y a là, de l'aveu des indigènes, un interdit très puissant, que le dernier des mécréants lui-même se sent très peu porté à enfreindre, pour ne pas encourir la vengeance de la tâd'a, particulièrement implacable, assure-t-on, dans ce cas'. On remarquera au passage que ces interdits font des parents de lait quelque chose de tout à fait différent des parents ordinaires. Cela montre bien le caractère 'construit' de la parenté de lait. Le pacte utilise de façon stratégique le langage de la parenté pour produire ses effets. La prohibition de l'inceste, comme en bien d'autres cas, est utilisée pour entretenir cette fiction.

[64] Quoiqu'il en soit, la transgression des interdits est sanctionnée par des amendes parfois très lourdes (lekhd'it) 'mais surtout l'horreur sacrée qui pousse à éviter un tel acte est entretenue par toute une foule de légendes qui font s'appesantir sur le malencontreux parjure non pardonné par son u-tâd'a, les plus terribles malédictions du ciel'. Marcy nous donne deux exemples de ces légendes. Dans la première, une femme partie avec ses enfants ramasser de l'herbe les fait monter au retour sur un âne qui lui semblait abandonné. En arrivant chez elle, elle ne peut plus descendre ses enfants désormais scellés sur la bête. A cette femme en pleurs des alliés de tâd'a apprennent que cette âne est le leur : 'O femme, apprends que le bien des Aït Tâd'a est sacré et qu'il ne faut jamais y toucher'. Une fois l'âne ramené, comme ils le lui demandent, à la place où elle l'a trouvé, elle peut enfin faire délivrer ses enfants prisonniers de leur monture'.

[65] La deuxième légende concerne un pacte de co-lactation entre deux douars Aït T'aleb Sa'id et Aït u-arab : 'Les Aït u-arab, à la recherche d'un amazir (emplacement) rencontrèrent celui que les Aït T'âleb Sa'id venaient d'abandonner. Ils décidèrent de s'y installer, ignorant qu'il était occupé par leurs Aït Tâd'a. II pleuvait (Marcy 1936 :973). Pour cuire le repas du soir les femmes ramassèrent hâtivement les débris de bois amassés par les premiers occupants. L'une d'elles posa un tapis sur la charge qu'elle venait de réunir ; aussitôt celui-ci pris feu. Les gens cherchèrent en vain, la cause matérielle de ce feu. C'est alors qu'ils pensèrent avoir commis l'erreur de s'installer sur l'amazir de leurs Aït Tâd'a. Ils abandonnèrent leur charges de bois et dès lendemain la djema'a se rendit chez les Aït T'âleb Sa'id. Elle raconta l'événement, s'excusa et demanda l'autorisation de se 
Citation: Vernier, Bernard (2006) 'Du bon usage de la parenté construite avec des humeurs corporelles (sang et lait) et quelques autres moyens', European Journal of Turkish Studies, Thematic Issue $\mathrm{N}^{\circ} 4$, The social practices of kinship. A comparative perspective, URL : http://www.ejts.org/document623.html

To quote a passage, use paragraph (§)

fixer sur leur ancien emplacement. Elle leur fut facilement accordée ; les femmes reprirent le bois abandonné et rien de fâcheux ne se reproduisit'. II est dommage que l'auteur n'ait pas recueilli de légendes portant sur la transgression d'interdits plus graves comme celui de l'inceste et celui du meurtre. L'horreur sacrée dont il parle suggère que la mort physique ou sociale pouvait faire partie des sanctions surnaturelles automatiques.

\section{Altorki et Khatib-Chahidi}

[66] Dans un article novateur qui porte sur les interdits de parenté de lait en Arabie Saoudite Altorki (1980) note que les ethnologues ont beaucoup travaillé sur la fraternité de sang (peut être parce qu'elle est masculine) et très peu sur la parenté de lait qui s'établit par les femmes et souligne les liens de parenté utérine. Elle rappelle que les lois islamiques reconnaissent la parenté par le sang (nasab), par l'alliance (musahara) mais aussi par le lait (rida'a). Ces trois types de parenté entrainent des interdits sexuels et de mariage. Pour la parenté de lait, les interdits dans le Coran sont très limités (mère et sœur de lait) mais, par un hadith, ils sont alignés sur ceux qui concernent la consanguinité (nasab). II faut, en outre, tenir compte de l'idée selon laquelle le lait d'une femme est provoquée par les relations sexuelles qu'elles a avec son partenaire d'où l'expression 'le lait de l'étalon'. De ce fait, une série d'interdits existent aussi entre les enfants de lait de la femme et la parenté du coté de son mari. Une transgression du tabou est censée produire les mêmes malheurs qu'en matière d'inceste ordinaire (maladie ou mort des enfants nés dans la relation coupable). Tous les observateurs sont d'accord pour dire que la parenté de lait produit une profonde affection entre parents et notamment, comme on le voit pour le prophète lui-même, dans son rapport avec sa nourrice entre une femme et l'enfant qu'elle a allaité.

[67] Les femmes qui habitaient ensemble avaient coutume d'allaiter les enfants les unes des autres (Altorki 1980 : 240). Une belle-mère ou une co-épouse pouvait allaiter l'enfant de celle qui ne pouvait le faire pour une raison médicale (son lait était mauvais etc.) ou qui, à la suite d'une dispute avec son mari, était retournée chez elle. On pouvait aussi demander à une esclave de donner le sein aux filles de la maison. De cette façon, celles-ci devenaient les sœurs de lait des fils de l'esclave. Elles n'avaient plus à se voiler devant eux ce qui rendait leur vie domestique plus facile. Les hommes mariés avaient souvent (ce qui était permis par la loi) une esclave comme concubine. Comme ils 
Citation: Vernier, Bernard (2006) 'Du bon usage de la parenté construite avec des humeurs corporelles (sang et lait) et quelques autres moyens', European Journal of Turkish Studies, Thematic Issue $N^{\circ} 4$, The social practices of kinship. A comparative perspective, URL : http://www.ejts.org/document623.html

To quote a passage, use paragraph (§)

étaient censés être à l'origine de la lactation, les enfants de la concubine devenaient germains de lait des enfants qu'ils avaient par mariage. On pourrait ajouter que la création d'une parenté de lait avec le personnel domestique devait contribuait à créer un attachement affectif entre maîtres et serviteurs ou pour le dire autrement à 'enchanter' les rapports nus de domination.

[68] On pouvait aussi utiliser cette stratégie de l'allaitement pour empêcher un mariage indésirable entre parents. II suffisait par exemple qu'une femme allaite les enfants du frère de son mari pour rendre impossible le mariage préférentiel avec la cousine parallèle patrilatérale, devenue la sœur de lait de ses cousins. Pour obtenir le même résultat, un homme pouvait demander à l'une de ses sœurs d'allaiter ses enfants et ceux de son frère. La parenté de lait était ici clairement utilisée pour manipuler la parenté en transformant un mariage possible (et même 'préférentiel') en mariage impossible. Elle avait l'avantage de fournir une raison acceptable par tous et qui, épargnant l'amour propre, permette d'éviter une brouille familiale. Cette habile 'gestion des relations intra-familiales' permettait de 'réconcilier préférence individuelle et attentes traditionnelles d'une façon non offensante' (1980 :243). Les femmes devaient y trouver une source de pouvoir. On pourrait ajouter que l'importance qu'a prise chez les théologiens - juristes musulmans la discussion autour du nombre de fois où l'enfant devait être allaité (une, cinq, dix ou quinze fois) et aussi celle, surréaliste, sur le mode d'absorption du lait (bouche, nez, oreille, yeux, anus) pour que la parenté de lait soit reconnue (il fallait théoriquement que ce lait ait contribué à la formation de sa chair et de ces os, mais dans quelle proportion?) suggère que l'utilisation stratégique de l'allaitement ne devait pas être rare. Et les femmes n'étaient-elles pas les mieux placées par leur mode de vie pour se livrer à ce type de manœuvre? Les docteurs ont décidé généralement que la parenté par allaitement ne pouvait être créée après l'âge de deux ans. Comme le note cette fois Gilali Avner, cette décision est importante, car une des stratégies féminines pour empêcher un mari d'avoir des relations avec une jeune esclave était d'allaiter celle-ci.

[69] Khatib-Chahidi (1981) attire l'attention sur le fait qu'alliance de mariage et parenté de lait pouvaient jouer le même rôle pour créer des interdits. En Iran chiite, chez les personnes dévotes on transforme les servantes et les serviteurs en personnes interdites en les mariant par des mariages temporaires à un enfant de la maison. Le domestique devient alors l'équivalent d'un beau fils ou d'une belle-fille pour le chef de la famille et une belle-sœur ou beau-frère pour ses enfants. Les femmes de la maison n'avait plus besoin de se voiler strictement, ce qui permettait aux domestiques 
Citation: Vernier, Bernard (2006) 'Du bon usage de la parenté construite avec des humeurs corporelles (sang et lait) et quelques autres moyens', European Journal of Turkish Studies, Thematic Issue $\mathrm{N}^{\circ} 4$, The social practices of kinship. A comparative perspective, URL : http://www.ejts.org/document623.html

To quote a passage, use paragraph $(\S)$

leur d'être plus efficaces dans leur travail. Mais la parenté de lait, malgré les interdits sexuels et matrimoniaux qu'elle produit, n'entraîne dans les pays musulmans aucun droit à l'héritage, ni aucune obligation légale d'assistance (109) et ne constitue pas une excuse en cas de vol (Hamilton 1791, II : 99, cité par Khatib-Chahidi).

\section{Gélard}

[70] Marie-Luce Gélard (2003, 2004), à qui je dois les références au travail de Marcy, attire l'attention sur l'usage du lait (agho) dans les stratégies d'alliance et de protection des Aït Khebbach du sud-est marocain. Cette tribu est divisée en plusieurs fractions appelé ighs (os/noyau). Les quatre fractions fondatrices endogènes se disent issues de quatre frères de même père. Elles monopolisent les fonctions de commandement (chef de la tribu et cheiks). Les fractions allogènes ont été intégrées par des pactes de protection sacralisés par le sacrifice d'un mouton et on les désigne d'ailleurs comme 'ceux qui ont sacrifié / égorgé': 'le groupe agrégé meurt à sa généalogie et renaît dans une autre fraction (l'os) à laquelle il appartient désormais'. Un repas commun scelle l'accord :'Et si un des individus qui a ainsi souscrit au pacte vient à manquer à ses engagements, on dit de lui : 'Dieu et la nourriture le lui rendront '(2004:16). La malédiction viendra donc de Dieu, mais aussi de la nourriture absorbée.

[71] Mais il existe aussi des pactes de co-lactation qui lient entre elles certaines fractions allogènes et dans certains cas même une fraction allogène à une fraction endogène. Ils se sont donc dans ce cas liés par le sang (sacrifice) puis par le lait.

[72] Les groupes liés par des pactes de co-lactation se considèrent comme des frères de lait. II existe entre eux un interdit sexuel et matrimonial appelé tafergant. Toute transgression est suivie d'une sorte de malédiction (maladie, paralysie, mort) qui s'abat sur le couple, leurs parents, leur descendance (enfants anormaux) ou sur les ressources familiales (dromadaires, bovins etc.). Comme l'avait signalé Marcy (1941), dans l'un des pactes de co-lactation, les femmes des deux tribus échangeaient les enfants qu'elles allaitaient, et ce pacte était appelé Tafergant du nom de l'interdit. La tradition orale mentionne un important pacte de paix avec une tribu arabe (2004: 20) : 'Avant, il y a eu une guerre à Timimoun et c'est là que quarante femmes des Aït Khebbach ont perdu leur mari en Algérie. Ce sont les hommes de la tribu qui ont décidé de leur construire une kasbah et 
Citation: Vernier, Bernard (2006) 'Du bon usage de la parenté construite avec des humeurs corporelles (sang et lait) et quelques autres moyens', European Journal of Turkish Studies, Thematic Issue $\mathrm{N}^{\circ} 4$, The social practices of kinship. A comparative perspective, URL : http://www.ejts.org/document623.html

To quote a passage, use paragraph (§)

de leur donner de la nourriture. Avec ces quarante femmes il y en avait autant des Beni M'Hamid. On a alors pris le lait des femmes des Aït Khebbach et celui de celles des Beni M'Hamid et on les a mélangés. Les bébés l'ont bu et c'est comme ça que tous les enfants sont devenus frères. C'est pour nous, un symbole de fraternité pour qu'il n'y ait pas de trahison'. Depuis, il existe une alliance permanente entre les deux groupes.

[73] Une autre tradition parle d'un pacte de lait 'réalisé entre les chefs de deux tribus (Les Aït Beggach avec une tribu arabe) ayant simplement bu du lait de chamelle à la même coupe laquelle, enterrée sur le lieu même de l'accord, a scellé définitivement le pacte d'alliance'.

[74] Dans cette société, le lait est censé posséder des pouvoirs magiques (il protège par exemple les enfants contre les djnûn). Quand on jure 'sur le lait fort' on encourt, en cas de parjure une vengeance mortelle. Et il est plus lourd de conséquence de jurer de cette façon que de jurer sur dieu. Dieu peut pardonner mais pas le lait (2004:22). L'importance de la parenté de lait, compréhensible dans les économies basées sur l'élevage nomade se voit ici dans des expressions comme 'le lait est plus fort que le sang' ou 'il y a le lait entre nous '. Gélard note que 'Si l'on peut 'couper le sang' c'est-à-dire, supprimer une communauté d'appartenance entre individus ou entre groupes, il est impossible d'annuler les liens indéfectibles de la parenté de lait'.

[75] Bruno et Bousquet (1946 :354, cités par Gélard 2004 :18) notaient que la parenté de lait peut être aussi utilisée pour demander la protection d'une femme sans même boire son lait : 'Il se rend à la tente de celui dont il demande la protection et se précipite au sein d'une des femmes de sa famille', c'est-à-dire 'je suis ton enfant, prends moi sous ta protection'. Chez les Aït Khebbach de Gélard, il suffit qu'un adulte ingère un peu du lait maternel recraché par l'enfant pour créer une parenté de lait entre l'enfant et cet adulte, parenté génératrice de prohibition matrimoniale. C'est d'ailleurs la raison pour laquelle les enfants encore au sein ne sont pas autorisés à boire dans le récipient collectif. On a trop peur qu'il recrache dans ce récipient ne serait-ce qu'une 'infime portion du lait maternel'.

[76] La naissance de l'interdit entre une fraction endogène (les Aït Amar) et allogène (les Aït Lahcen) est particulièrement intéressante (Gélard 2004 :61-62). Elle n'est pas fondée sur un pacte de lactation, mais sur une dispute entre les deux groupes. Un des membres de la famille des Aït Amar aurait volé une chèvre et l'aurait mangée lors d'un repas auquel avait été convié un homme de 
Citation: Vernier, Bernard (2006) 'Du bon usage de la parenté construite avec des humeurs corporelles (sang et lait) et quelques autres moyens', European Journal of Turkish Studies, Thematic Issue $\mathrm{N}^{\circ} 4$, The social practices of kinship. A comparative perspective, URL : http://www.ejts.org/document623.html

To quote a passage, use paragraph (§)

la fraction des Aït Lahcen. Comme le propriétaire de la chèvre volée réclamait que sa bête fut payée l'homme des Aït Amar demanda à son invité de payer la part qu'il avait consommée : 'L'homme des Aït Lahcen, furieux, se leva, et jurant de payer intégralement la chèvre (...) s'écria : 'Que Dieu balaie l'aire à battre de tous les Aït Amar qui épouseront une femme des Aït Lahcen et inversement'. Cette injonction signifiait que toute alliance entre eux 'serait stérile et préjudiciable aux époux'. II me semble que la création de cet interdit peut s'expliquer dans la logique de la théorie de l'inceste que je défends. Dans une société qui attache autant d'importance à l'hospitalité, la grossièreté de la demande des Aït Amar a déclenché une juste colère des Aït Lahcen qui appartiennent à la même tribu mais comme 'pièce rapportée' et en position dominée par rapport au groupe endogène. Le refus de l'alliance est le refus d'un certain type de rapport qui conduit à la fusion des deux groupes. Ce refus est compatible avec l'intérêt des Aït Lahcen de continuer à appartenir à la tribu. Le modèle de cette appartenance à distance est fourni par les relations entre les groupes de la même tribu et qui sont liés par la parenté de lait. La décision de l'Aït Lahcen nous livre la vérité des autres interdits basés eux sur un pacte de co-lactation. Et n'est-il pas clair que les inter-mariages entre fractions désormais ennemies sont menacés (tant que la plaie n'est pas cicatrisée et qu'une nouvelle décision d'alliance, qui peut très bien prendre la forme d'un mariage solennel, n'est pas prise) par des difficultés relationnelles entre conjoints, chacun pouvant reprocher à l'autre son appartenance de fraction?

\section{Peter Parkes}

[77] Peter Parkes a mené une recherche bibliographique impressionnante sur la parenté de lait. II a offert récemment au lecteur $(2001,2003,2004)$ une foule de données comparatives passionnantes que nous allons utiliser ici. L'anthropologue part du travail d'Hammel (1968) qui avait constaté que les groupes agnatiques hiérarchisés dans la région des Balkans (Serbie) qu'il étudiait étaient reliés par une chaîne de relations de parrainage (Kumstvo) asymétriques ou unilatérales (analogue à l'une des formules du mariage kachin analysé par Leach où les femmes circulent de haut en bas des groupes sociaux). Ici ce sont les enfants qui jouent le rôle de créateur d'alliance (Goody et surtout Lallemand) en circulant vers le bas : A parraine quelqu'un du groupe inférieur B qui parraine quelqu'un du groupe inférieur $C$, etc.). Cette chaîne de relation de parenté spirituelle tend à 
Citation: Vernier, Bernard (2006) 'Du bon usage de la parenté construite avec des humeurs corporelles (sang et lait) et quelques autres moyens', European Journal of Turkish Studies, Thematic Issue $N^{\circ} 4$, The social practices of kinship. A comparative perspective, URL : http://www.ejts.org/document623.html

To quote a passage, use paragraph (§)

se reproduire d'une génération à l'autre. Filiation, alliance et parenté spirituelles sont des structures sociales alternatives. Les groupes agnatiques pouvaient établir des relations aussi bien par la parenté spirituelle que par le mariage. Parkes fait progresser le travail de généralisation commencée par Hammel en incluant dans son modèle la parenté de lait. II montre qu'en Eurasie le fosterage ou plus précisément la parenté de lait peut être substituée au parrainage comme structure sociale alternative et forment souvent aussi ce genre de chaîne unilatérale. II existe donc un 'isomorphisme structural' entre les deux instituions. Ce sont aussi les enfants confiés à une nourrice qui circulent vers le bas. Sa lecture de l'étude de Biddulph (1880) sur les royaumes montagneux du Hindu Kush où, comme on l'a vu plus haut, les enfants des familles régnantes étaient envoyés très tôt après leur naissance dans des familles nobles pour y être allaités et élevés, nous apporte de nouveaux renseignements. Biddulph, pour qui la parenté de lait était, dans le Hindu Kush plus importante que la parenté de sang souligne que les histoires des dynasties locales abondent en trahisons et meurtres (fils contre père et frère contre frère). De ce fait, la parenté de lait offrait une alternative affective parfois littérale (du fait de la mort du père ou du frère) à la parenté de sang. C'est ce qu'indique clairement Leitner (1894): 'Un parent de sang dans les familles dominantes est une personne que Dieu désigne à quelqu'un comme un obstacle sur sa route qu'il faut tuer tandis qu'un parent foster (généralement de basse classe) est un véritable ami qui s'élève et s'abaisse avec soi'. Dans les luttes dynastiques, les prétendants s'affrontaient avec chacun, comme supporteurs, ses parents de laits qui entraînaient avec eux le soutien de leur groupe d'appartenance (familles ou clans). Les prétendants qui perdaient étaient massacrés ou partaient en exil avec leurs parents de lait. La parenté de lait servait aussi pour se protéger de l'adultère : 'En cas (d'infidélité) une garantie est prise pour le futur en plaçant les lèvres de l'accusé sur la poitrine de la femme. Elle est alors regardée comme sa foster-mother et aucune autre relation que celle mère/fils ne peut exister entre $\operatorname{eux}(2001: 11)$.

[78] Selon Peter Snoy (1975) il existait une fraternité de lait inter-régionale entre certaines enclaves de l'ethnie Shin comme collectivement alliée avec Hunza et une confraternité rivale qui regroupait d'autres enclaves avec apparemment des interdits d'intermariage entre les alliés foster. Les archives de Hussam ul Muk montre qu'à Chitral on dispersait volontairement les princes et princesses de même père et mère dans des familles subordonnés rivales des différentes régions et dont le statut dépendait en partie de celui de la mère de l'enfant (femme/concubine). Les postes 
Citation: Vernier, Bernard (2006) 'Du bon usage de la parenté construite avec des humeurs corporelles (sang et lait) et quelques autres moyens', European Journal of Turkish Studies, Thematic Issue $N^{\circ} 4$, The social practices of kinship. A comparative perspective, URL : http://www.ejts.org/document623.html

To quote a passage, use paragraph (§)

administratifs convoités comme wazir ou atalek (père de lait) étaient des 'dépouilles de guerre' que les parents de lait des princes rivaux ensemble 'avec leur réseau de 'parents'dépendants' se disputaient car ils permettaient de contrôler les revenus du royaume. Le père de lait occupait le poste de trésorier d'Etat. Ici aussi, la parenté de lait établissait des relations unilatérales entre ordre de statut $(2001: 17)$ et prenait dans ses mailles l'ensemble des groupes de statut. Elle créait des liens de parenté plus solides que l'alliance (on pouvait divorcer) ou que la filiation (on pouvait la désavouer) et qui se reproduisaient, comme la parenté spirituelle dans les Balkans, de génération en génération. Ici aussi, la trahison était le fait de consanguins ou d'affins et non de parents de lait.

[79] II existait aussi une forme d'allaitement collectif. Schomberg écrit en 1938 : 'II n'est pas rare pour un enfant de faire le tour de tout un village ou tribu bénéficiant du lait de plusieurs douzaines de mère différentes (...).Cela agrandissait le réseau protecteur de relations de lait de l'enfant une fois grand' (2001:19). De même au Daguestan, quand un fils naissait dans la famille gouvernante, il était envoyé de village en village pour être allaité par toutes les femmes possibles pour le rendre germain de lait de toute sa génération (Chenciner 1997). C'était une façon d'assurer le maximum de personnes au bien être de l'enfant et de lui procurer des alliés pour les luttes dynastiques qui, s'il arrivait au pouvoir, pouvaient bénéficier de postes officiels. Un fils de Shahzada Hussam-ul-Mulk aurait eu environ 150 mères provenant d'une section tribale où il a été plus tard affecté comme gouverneur. La chaîne unilatérale de parents de lait semble avoir descendu jusqu'au serf lui-même et s'être accompagnée d'échanges, de dons cérémoniels (2001:20). Le seigneur propriétaire de la terre fournissait un ensemble d'habits fins au mariage de la fille de son parent de lait et, en échange, il se réservait une partie de la compensation matrimoniale donnée par la famille du mari (cheval ou taureau). En Abkhazie (Inal-Ipa 1956, Grigolia 1962, cités par Parkes), les enfants (garçons ou filles) étaient confiés à un père de lait (Atalik) appartenant à une famille plus basse socialement. Le lien entre les deux familles était considéré comme plus sacré que le lien de consanguinité. II s'étendait aux groupes patronymiques avec interdit de mariage sur au moins sept générations. En temps de guerre l'enfant de la famille 'adoptive' avait le devoir de protéger son frère de lait avec son propre corps. Cette parenté de lait qui semble indépendante de l'islam, car elle existait aussi chez les Caucasiens chrétiens, entraînait des prohibitions de mariage aussi étendues que pour la parenté de sang. Un allaitement symbolique (akukatshara: mordiller la poitrine) était aussi utilisé dans la cérémonie pour faire la paix après les combats inter-tribaux. II s'agissait parfois 
Citation: Vernier, Bernard (2006) 'Du bon usage de la parenté construite avec des humeurs corporelles (sang et lait) et quelques autres moyens', European Journal of Turkish Studies, Thematic Issue $N^{\circ} 4$, The social practices of kinship. A comparative perspective, URL : http://www.ejts.org/document623.html

To quote a passage, use paragraph (§)

pour deux hommes (et c'est vrai aussi en Irlande) de se mordiller leur mamelon masculin. C'est dire le caractère essentiellement métaphorique de la parenté de lait (2004 :591). Les Abkhazes pensaient qu'il n'y a pas de pouvoir plus fort que le lait de la mère pour nettoyer le sang de la vengeance. Celui qui mélange le sang au lait est mis hors la loi par la famille et la parenté, 'tuer était considéré comme un affront à la poitrine sacrée, une profanation blasphématoire de sa poitrine [car] le lait du sein de la mère était vu comme un symbole d'absolue pureté et de sainteté doté d'un pouvoir immense et sans prix qui a la propriété de brûler tous ceux qui transgressent sa sainteté'. On aurait réussi à ne mettre fin à une guerre intertribale légendaire qu'en apportant cinq cents femmes qui allaitaient avec leurs bébés dans les bras pour effectuer un échange collectif d'enfants en vue d'une 'adoption' réciproque (2003 :345). Les femmes auraient déclaré : 'mon sang (c'est-à-dire le lait de ma poitrine) pardonne pour le sang de mon fils'. Ce rite de morsure de la poitrine était un rite d'allégeance pratiqué en Géorgie par le chevalier servant qui se dévouait à une femme noble. Mais c'est tout un clan ou une tribu qui pouvait aussi faire allégeance au roi par l'allaitement collectif de rejetons royaux. Filipovic raconte qu'en Bulgarie quelqu'un pouvait dire 'donne moi une goutte du lait de ta mère pour que je ne puisse pas être ton ennemi' ou 'buvons ensemble le lait d'une mère pour que nous puissions vivre comme des frères dans ce monde '. Dans le sud de la Bulgarie (Rhodopes) la parenté de lait était supposée s'étendre sur douze générations.

[80] Parkes note aussi que l'étude comparative de Gwynn montre l'existence en Eurasie d'une stratégie dynastique récurrente qui utilise le fosterage comme mécanisme d'allégeance qui lie gouvernants et subordonnés (tribus, clans, familles ou groupes ethniques différenciés) et où les deux parties en retirent des avantages de différents types (économique, politique, symbolique etc.) selon leur position dans ces rapports. On retrouverait des structures analogues au pays de Galles en Irlande, en Islande et travers la Turquie, mais aussi dans toute l'Asie islamique et dans l'île Gilbert en Océanie où Lambert (1964) compare déjà la circulation asymétrique d'enfants, du plus haut chef aux villageois, à la circulation des femmes chez les Kachin. A la succession du chef, des querelles éclataient aussi entre les différents prétendants et leurs alliés de lait. A propos du pays de Galles, Parkes cite ce texte $(2003: 755)$ de Giraldus Cambrensis, dont le contenu nous semble désormais familier : 'une sérieuse cause de dissensions est l'habitude des princes du pays de Galles de confier l'éducation de chacun de leur fils à différents nobles vivants sur leur territoire. Si le prince meurt chaque noble complote et s'efforce d'assurer la succession au profit de l'enfant qu'il a élevé (foster - 
Citation: Vernier, Bernard (2006) 'Du bon usage de la parenté construite avec des humeurs corporelles (sang et lait) et quelques autres moyens', European Journal of Turkish Studies, Thematic Issue $N^{\circ} 4$, The social practices of kinship. A comparative perspective, URL : http://www.ejts.org/document623.html

To quote a passage, use paragraph $(\S)$

child) et d'être sûr qu'il sera préféré aux autres frères. Le résultat est que les plus effrayants désordres se produisent dans leurs territoires les gens étant assassinés, les frères se tuent les uns les autres et même s'arrachent les yeux les uns aux autres. .. II s'en suit que vous trouverez que l'amitié est beaucoup plus chaude entre frère de lait qu'entre vrais frères'. II cite aussi Topographia Hibernica pour souligner que les Irlandais ont des opinions semblables, 'qui, préférant leur enfant d'adoption et leur frère de lait, persécuteront leur propres frères et parents'.

[81] Parkes (2003) souligne d'après une remarque de Goitein que la parenté de lait pouvait parfois légitimer une succession dans les premiers califats de l'islam : 'll est bien connu que Harun al-Rashid a été allaité par une femme de la famille Barmak (...), tandis que Al-Fadl le Barmecide était allaité par la future mère du caliphe. Un exemple de relation de lait entre Abassides et Barmecides est noté par Tabary'.

[82] L'allaitement collectif (étendu au clan ou à la tribu) est aussi attesté au Vlème en Irlande et crée souvent un lien d'amitié et d'alliance entre deux ou plus tribus, et même entre provinces. Enfin, pour revenir en Europe, Nelson (1999) raconte que dans l'Aragon espagnol du Xlème siècle le jeune roi quand il accède au trône confie à son frère de lait la position de chef conseiller et il appelle ses frères et sœurs fictifs à la cour, comme alliés de confiance.

[83] Dans un récent article (2004) Parkes souligne la contradiction de la pensée populaire qui, à travers ses dictons, tantôt affirme que la parenté de lait (ou de baptême) est la parenté la plus importante, tantôt dit la même chose de la parenté ordinaire 'par le sang'. Il y voit le signe que la représentation de la parenté fictive est marquée par une ambivalence morale qui oscille entre idéalisation et dévalorisation. L'appartenance à plusieurs types de réseaux de parenté crée une hésitation entre plusieurs loyautés. On peut préférer, on l'a vu, les membres de sa famille de lait à ceux de sa vraie famille et même avoir de l'aversion pour ces derniers et leur nuire. Dans l'alliance de parenté où l'enfant de milieu supérieur est confié à des parents d'une couche sociale plus basse, les parents d'adoption peuvent être amenés à négliger leurs propres enfants. Surtout, comme cette relation relie des groupes sociaux inégaux, sans mariage possible, elle revêt un caractère non naturel perçu comme tel. C'est souvent du clientélisme politique 'habillé du faux plumage de la parenté' (2004 : 606). La notion de mutuelle assistance peut recouvrir en réalité une dissymétrie dans le respect des obligations. Le seigneur peut être ingrat envers ses parents de lait et les abuser 
Citation: Vernier, Bernard (2006) 'Du bon usage de la parenté construite avec des humeurs corporelles (sang et lait) et quelques autres moyens', European Journal of Turkish Studies, Thematic Issue $\mathrm{N}^{\circ} 4$, The social practices of kinship. A comparative perspective, URL : http://www.ejts.org/document623.html

To quote a passage, use paragraph (§)

(ajoutons: ou l'inverse subversif). II n'est pas rare que les légendes (par exemple abkhasiennes, mais aussi irlandaises) racontent des histoires de trahison, abus de confiance, adultère ou inceste entre parents de lait où le seigneur abuse de sa position de force. Entre seigneurs il est plus commode de se faire payer une dette de sang sur la personne du père ou du frère nourricier du meurtrier. Finalement, 'bien que pour tous les partenaires cette parenté adoptive pouvait être un idéal romantique attrayant d'allégeance réciproque et d'amitié d'honneur qui transcendent les différences de statuts (...) en pratique' (2004 : 608), cette parenté adoptive idéale ne pouvait jamais être réalisée de façon aussi satisfaisante comme relation entièrement comparable et compatible avec la parenté de naissance.

\section{Parenté de lait, de sang, de parrainage, de baptême et de circoncision}

[84] Une des grandes leçons que je retiens de Bourdieu est que l'analyse d'un fait ethnologique doit expliciter l'anthropologie de l'action qu'elle met en œuvre, si elle veut se donner tous les moyens d'être scientifique. Ce conseil est particulièrement pertinent. II permet d'observer que Lévi-Strauss (et l'on pourrait dire la même chose de certains de ses disciples les plus illustres) a développé ses analyses à partir d'une anthropologie de l'action non consciente d'elle-même et donc tentée d'être à géométrie variable en fonction des besoins démonstratifs du moment. II explique, par exemple, les pratiques matrimoniales et la prohibition de l'inceste par les structures inconscientes de l'esprit, mais ailleurs le mariage frère/sœur chez les pharaons par des raisons plus prosaïques comme l'intérêt dynastique. Je placerai mon analyse, ici comme d'autres fois, dans la cadre d'une anthropologie globalement bourdieusienne pour qui 'la parenté est quelque chose que l'on fait et dont on fait quelque chose'13.

[85] 1- Toutes les parentés ordinaires et 'fictives' (de lait, de sang, etc.) y compris la parenté baptismale peuvent être utilisées pour des fins légales ou non légales.

[86] 2- La parenté de lait et de sang sont toutes deux capables, comme l'alliance matrimoniale mais aussi la parenté baptismale (en Serbie notamment) et le parrainage de circoncision (Magnarella et Turkdoğan 1973), de lier des individus entre eux (parents, voisins etc.) ou 
Citation: Vernier, Bernard (2006) 'Du bon usage de la parenté construite avec des humeurs corporelles (sang et lait) et quelques autres moyens', European Journal of Turkish Studies, Thematic Issue $N^{\circ} 4$, The social practices of kinship. A comparative perspective, URL : http://www.ejts.org/document623.html

To quote a passage, use paragraph (§)

des groupes et même d'établir une alliance organique et se reproduisant d'une génération sur l'autre entre groupes (familles, lignages, clans et au moins pour le lait et le sang, tribus, ou même villages, régions, nomades et sédentaires, etc.) en proposant alors des formules globales d'intégration et /ou en opposant les pactes de paix à l'état de guerre ou de vendetta.

[87] C'est dire les limites de l'analyse que fait Lévi-Strauss de la 'fraternité de sang' dans Les structures élémentaires de la parenté (pp.554 -555). Après avoir remarqué qu'elle crée bien, elle aussi, un lien d'alliance entre les individus, il ajoute, qu'à la différence de l'alliance par le mariage, elle assimile les intéressés à des frères et à des sœurs avec qui il est interdit de se marier. II en résulte une grande différence entre le lien de fraternité et d'alliance: 'I'un constate une solidarité mécanique (frère), tandis que l'autre invoque une 'solidarité organique' (beau-frère, ou compère). Les frères sont proches les uns des autres, mais ils le sont par leur similitude, comme les poteaux ou les tuyaux des flûtes (chez les indiens Nambikwara 'les objets qu'on rencontre sous forme de série, tels que les poteaux de la hutte, les tuyaux de la flûte de pan etc., sont dits 'frères' les uns des autres') ; au contraire, les beaux-frères sont solidaires parce 'qu'ils se complètent et possèdent, l'un pour l'autre, une efficacité fonctionnelle; soit qu'ils jouent le rôle de l'autre sexe dans les jeux érotiques de l'enfance, ou que leur alliance masculine, à l'âge adulte, se sanctionne par la fourniture à chacun de ce qu'il ne possède pas : une épouse (grâce à la renonciation simultanée à ce que l'un et l'autre détiennent), une sœur. La première forme de solidarité n'ajoute rien, n'unit rien ; elle se fonde sur une limite culturelle, qui se satisfait par la reproduction d'un type de connexion dont la nature fournit le modèle ; l'autre réalise une intégration de groupe sur un nouveau plan' Et à propos du travail de Ralph Linton sur la fraternité de sang aux Marquises (la relation enoa) il observe que la fraternité de sang 'n'est qu'une solution individuelle jouant le rôle d'un substitut, lorsque la solution véritable et efficace des relations entre les groupes, c'est-à-dire la solution collective et organique des intermariages avec fusion consécutive des tribus, est rendue impossible par la situation internationale: bien que des vendetta soient en cours, l'institution des enoa, affaire purement individuelle, peut assurer un minimum de liaison et de collaboration, alors même que le mariage, qui est affaire de groupe, ne peut opérer (...)'.

[88] Or dès 1935, bien avant la publication du livre de Lévi-Strauss, Brenda Seligman avait contesté le fait que la femme 'soit l'instrument unique ou prédominant de l'alliance' en invoquant' l'institution de la fraternité de sang, telle que celle exprimée par la relation de henamo chez les 
Citation: Vernier, Bernard (2006) 'Du bon usage de la parenté construite avec des humeurs corporelles (sang et lait) et quelques autres moyens', European Journal of Turkish Studies, Thematic Issue $N^{\circ} 4$, The social practices of kinship. A comparative perspective, URL : http://www.ejts.org/document623.html

To quote a passage, use paragraph (§)

indigènes de la Nouvelle-Guinée' (Seligman 1935). En 1939 en France même, Denise Paulme avait déjà également analysé ce type de pacte comme une des façons, parallèlement au mariage, de contracter une alliance. De façon générale, les travaux des ethnologues ont amplement prouvé que, sur cette question, Lévi-Strauss s'était trompé. Comme les ethnologues l'ont remarqué, ce qu'on appelle improprement la 'fraternité de sang' peut créer entre les groupes une relation qui n'est ni assimilable à une fraternité consanguine ni à une relation d'alliance matrimoniale, mais qui (il faudrait ajouter : à côté d'autres formes de parenté comme par exemple les simples rapports de parenté à plaisanterie sans tabou sexuels et matrimoniaux) n'en instaure pas moins une véritable solidarité organique. L'importance fonctionnelle de cette 'fraternité' est clairement soulignée par le fait que ceux qui transgressent les règles de comportement entre frères sont condamnés à une mort certaine. Dans la région de Jos au Nigéria elle est, on l'a vu, dans certains domaines, plus grande que la parenté par alliance. La relation Apari crée un réseau de relations sociales extrêmement dense et complexe. Elle contribue à instaurer une coopération entre les membres des groupes et des sousgroupes et à établir les conditions de la paix entre les groupes, entre les sous-groupes et à l'intérieur de chacun d'entre eux (la "fraternité de sang" créée des liens organiques entre groupes et pas seulement entre individus).

[89] On voit que l'échange des femmes n'est pas au fondement de l'existence des sociétés. Les relations d'alliance matrimoniale sont si peu au fondement de l'existence des sociétés que dans de nombreuses sociétés les relations entre groupes se nouent non par l'intermariage mais par son contraire : l'interdit de se marier et d'avoir des relations sexuelles ${ }^{14}$. II y a donc, au moins, deux façons opposées de créer une alliance entre groupes, de pacifier leurs rapports et d'instaurer l'entraide en utilisant la sphère sexuelle et matrimoniale. L'une, plus courante et sur laquelle les ethnologues ont concentré leur attention, est l'intermariage. L'autre, que les ethnologues ont parfois négligé est l'alliance par les interdits sexuels. Ces deux solutions produisent des effets qui leur sont propres et ont chacune leurs avantages et leurs inconvénients.

14 J'ai examiné ailleurs (Vernier 1999) les autres raisons qui invalident la proposition de Lévi-Strauss. Beaucoup d'ethnologues ont décrit cet interdit entre groupes étrangers sans toujours en tirer les conclusions nécessaires. Ils continuent, de ce fait, à répéter, sans la remettre en question, une thèse attribuée à Lévi-Strauss et qui n'offre en réalité qu'une version mutilée d'un complexe d'idées, plus riche, que l'on trouve chez les pères de l'Eglise et tout spécialement chez Saint Augustin. 
Citation: Vernier, Bernard (2006) 'Du bon usage de la parenté construite avec des humeurs corporelles (sang et lait) et quelques autres moyens', European Journal of Turkish Studies, Thematic Issue $N^{\circ} 4$, The social practices of kinship. A comparative perspective, URL : http://www.ejts.org/document623.html

To quote a passage, use paragraph (§)

[90] 3- Ces parentés dites fictives sont susceptibles d'autres utilisations politiques. La fraternité de sang peut donner au roi des conseillers ou des espions fidélisés par le sang. La fraternité de lait peut fournir de nombreux alliés (par allaitement collectif du prétendant par un clan ou une tribu). A Karpathos (Vernier 1991), les Canacares baptisaient de nombreux villageois et notamment des bergers pour avoir des alliés. En Grèce moderne, mais aussi ailleurs, les politiciens baptisent par dizaines les enfants de leurs électeurs pour obtenir des voix, les industriels baptisent les enfants de leurs ouvriers ce qui profite au bon fonctionnement des rapports de production. Le parrainage de circoncision permet, comme ailleurs le parrainage, un échange de services du type vote contre 'piston' (Sertel 1971).

[91] 4- Le parrainage de baptême ou de circoncision comme la parenté de lait et comme l'alliance matrimoniale peut avoir un caractère réciproque ou unilatérale (Le groupe $A$ baptise le groupe $B$ qui baptise $C$, etc.). Toutes ces parentés, comme la parenté de sang, peuvent lier des personnes ou des groupes qui sont socialement égaux ou socialement inégaux.

[92] 5- Les pratiques matrimoniales obéissent le plus souvent à des stratégies de reproduction et donc à une relative homogamie sociale. Du fait que la parenté est prédisposée par sa nature à occulter les rapports de domination, voire d'exploitation (Vernier 1980, 1984, 1991 et 1998) les dominants peuvent avoir avantage pour maintenir l'ordre social à sacrifier partiellement les bénéfices qu'ils retirent de l'homogamie en donnant (comme chez les Kachin. cf. Leach 1961) certaines de leurs femmes à leurs inférieurs (l'inverse n'est pas inconcevable ailleurs) pour se les concilier en en faisant des débiteurs dévoués à leurs intérêts. Mais si pour une raison liée aux caractéristiques de la structure sociale et/ou à leur représentation du monde social ils rechignent à mêler sexuellement des corps enracinés dans des positions sociales trop éloignées (ex :caste supérieure /inférieure, maître/ esclave, seigneur/serf etc.) et à obtenir des enfants de ce mélange, ils peuvent utiliser la parenté par le sang ou par le lait qui comme la parenté baptismale fournit un autre moyen d'enchanter les rapports de domination en les annulant fictivement et en les enrobant d'affectivité. De façon plus générale les ethnologues ont bien vu que ces parentés (y compris le parrainage de circoncision) pouvaient remplacer l'alliance matrimoniale quand celle-ci n'est pas souhaitée pour des raisons de différences d'ethnie ou de religion. II resterait à prendre en compte les cas ou même la parenté fictive est impossible et à observer alors les mécanismes alternatifs de domination ou de coexistence pacifique. 
Citation: Vernier, Bernard (2006) 'Du bon usage de la parenté construite avec des humeurs corporelles (sang et lait) et quelques autres moyens', European Journal of Turkish Studies, Thematic Issue $N^{\circ} 4$, The social practices of kinship. A comparative perspective, URL : http://www.ejts.org/document623.html

To quote a passage, use paragraph (§)

[93] 6- En même temps, les parentés par le sang ou le lait permettent, comme l'alliance quand elle se porte sur des non-parents et comme tous les types de parenté dite fictive, d'étendre le réseau de relations utiles, d'entraide, de solidarité, d'hospitalité dont chacun peut bénéficier.

[94] 7- La parenté de lait et de sang comme le parrainage baptismal ou de circoncision en Turquie (Sertel 1971) s'accompagnent souvent d'interdits d'inceste. C'est une des façons possible d'affirmer que ces parentés (comme la parenté par alliance dans le cas des interdits entre affins) sont aussi importantes que la proche parenté dite consanguine auquel on associe d'abord l'interdit. D'où souvent un alignement du champ d'application des interdits sur celui qui existe en matière de parenté ordinaire. Et d'où même, parfois comme dans le cas de certains pays musulmans, de multiples interdits de lait qui, les ethnologues l'ont remarqué, contrastent de façon troublante avec la possibilité du mariage entre cousins germains et de façon plus générale avec le petit nombre des interdits de parenté ordinaire.

[95] 8- Mais il existe d'autres moyens d'affirmer l'importance de la parenté fictive. Les Azandé et d'autres (pour des raisons qu'il reste à étudier) ont choisi eux de renforcer la 'fraternité de sang' par une alliance matrimoniale possible avec une parente de son 'frère'. De la même façon que l'on peut parfois renforcer les parentés de lait et de sang l'une par l'autre, comme le montre l'exemple examiné par Gélard, où des groupes commencent par un pacte de sang à base de sacrifice puis le renforce par un pacte de lait.

[96] 9- D'une certaine façon, la parenté dite fictive, y compris le parrainage, est condamnée (précisément parce qu'elle est fictive) à se donner à voir comme plus importante que les parentés consanguine et par mariage : pas de divorce et peu de possibilité de désaveu. Ce n'est donc pas un hasard si les transgressions des obligations (entraide etc.) et surtout des interdits (vol, inceste) conduisent souvent à une sanction automatique et plus forte (qui conduit facilement à la mort pour soi et ses proches), qu'une transgression en matière de parenté ordinaire. Cette importance est aussi liée bien sûr aux propriétés magiques des substances utilisées (lait, sang, huile sainte etc.) et au fait que ces parentés sont associées dans tous les cas à des rites à caractère 'religieux'.

[97] 10- Mais c'est aussi que la parenté par le sang et par le lait (comme la parenté baptismale chez Fine par exemple) sont, sous un certain rapport, réellement plus importante parce qu'elles comblent (et sont chargées de combler), chacune à sa façon, les manques ou les défauts 
Citation: Vernier, Bernard (2006) 'Du bon usage de la parenté construite avec des humeurs corporelles (sang et lait) et quelques autres moyens', European Journal of Turkish Studies, Thematic Issue $N^{\circ} 4$, The social practices of kinship. A comparative perspective, URL : http://www.ejts.org/document623.html

To quote a passage, use paragraph (§)

des deux parentés ordinaires (selon les cas : rapports inégalitaires, conflit d'intérêts autour de la compensation matrimoniale, de l'héritage ou du travail, rivalités affectives ou pour le pouvoir, mauvais traitement, contrôle des enfants etc.). Elles protègent aussi des menaces qui peuvent venir de ces parentés, tout spécialement de la parenté consanguine. Dans le cadre d'une société chrétienne pour qui le sexe est une chose impure et qui croit au paradis la parenté baptismale est là pour ôter la souillure du péché et remplacer les parents de chair par des parents spirituels mieux à même d'assurer le salut (par leurs conseils et leurs prières). L'Eglise utilise cette argumentation comme une des idéologies justificatrices de son pouvoir social. De leur côté, les fraternités de sang ou de lait peuvent fournir des substituts de parents en cas de mauvaises relations familiales. Surtout, elles peuvent fournir des alliés contre certains parents dont la haine structurale peut se manifester soit par la sorcellerie, souvent plus efficace entre parents (car une de ses fonctions est d'inciter chaque parent à remplir ses devoirs d'entraide), soit (dans le Hindu Kush) par l'assassinat des rivaux, fréquent dans les familles gouvernantes du fait de l'importance des enjeux ${ }^{15}$.

[98] Le lien par le sang (comme le lien par le lait, le parrainage de baptême et de circoncision) peut venir se surajouter au lien d'alliance (le sang d'un sacrifice) pour le sacraliser et par exemple rendre la femme ou les conjoints fidèles. II peut au contraire être établi en premier pour permettre une alliance sans friction avec un groupe étranger. La parenté de sang (comme dans le cas où des frères se font frères de sang pour faire créer une alliance indestructible contre un des leurs qui a usurpé le pouvoir) et celle de lait, comme la parenté de baptême ou de circoncision et comme l'alliance matrimoniale, peut venir renforcer la parenté consanguine en privilégiant les relations avec certains parents plutôt qu'avec d'autres. La parenté de sang et de lait, du fait de leur extrême importance, sont en position de réguler le fonctionnement des rapports de parenté ordinaire notamment en s'efforçant de prévenir les conflits. On peut essayer de préserver la relation conjugale en faisant de l'amant réel ou potentiel un frère de lait (ou un parrain de son enfant), ce qui interdit désormais toute relation sexuelle. Dans la même logique, la parenté de lait peut être utilisée pour transformer les membres du groupe domestique (consanguins, alliés et non parents, serviteurs ou esclaves) en parents de lait, évitant ainsi que les rivalités sexuelles, et la subversion de l'ordre social qui lui est liée, ôte à ce groupe sa fonctionnalité. On peut l'utiliser pour empêcher des mariages entre

15 Dans l'ancienne Egypte les prétendants au poste de pharaon s'entretuaient en famille. Utilisaient-ils une parenté ' fictive ' comme la parenté de lait pour trouver des alliés parmi les non parents ? Voici le genre de question que cette étude amène à poser à l'histoire. 
Citation: Vernier, Bernard (2006) 'Du bon usage de la parenté construite avec des humeurs corporelles (sang et lait) et quelques autres moyens', European Journal of Turkish Studies, Thematic Issue $N^{\circ} 4$, The social practices of kinship. A comparative perspective, URL : http://www.ejts.org/document623.html

To quote a passage, use paragraph (§)

certains parents. Comme l'ont bien vu les ethnologues, elle a l'avantage de fournir une raison qui n'est pas blessante. Elle satisfait donc les intérêts des stratèges tout en minimisant les risques pour l'intégration du groupe. Elle permet aussi aux femmes de vivre plus librement et fonctionnellement en leur permettant, dans les pays musulmans, de circuler et de travailler sans voile. Le frère de sang ou de lait est un médiateur privilégié dans les disputes entre conjoints (le mangou dogon et l'apari nigérian). II l'est aussi quand surviennent des disputes entre les membres du groupe allié (les mêmes que précédemment). Une parenté ordinaire peut donc être régulée par une parenté fictive. On peut ajouter que déjà la parenté par alliance matrimoniale avec des non parents, comme l'ont bien vu Freud et Malinowski, peut être envisagée, ceci est capital, comme un moyen (par la médiation du tabou de l'inceste) de réguler le fonctionnement de la famille en évitant les causes de conflit que provoque la rivalité sexuelle De même, l'interdit entre frères de sang ou de lait a aussi comme fonction d'éliminer une cause importante de conflit en supprimant la possibilité de rivalité au sujet des femmes.

[99] Ce travail de régulation des rapports d'alliance (entre époux) et des rapports de parenté est désormais entrepris par des services publiques ou privés (police, justice, éducateurs spécialisés, assistantes sociales, psychologues, psychanalystes) qui interviennent dans les divorces, les cas d'assassinat, de violence conjugale ou de 'maltraitance' d'enfants à la demande des intéressés (ou de certains d'entre eux) et qui, plus généralement, imposent dans la sphère privée les lois du pays. En ce sens, ces professionnels remplacent les frères de lait, de sang et autres parents 'fictifs'.

[100] 11- La nécessité de donner à la parenté fictive plus d'importance qu'à la parenté consanguine contient cependant une menace implicite pour la parenté ordinaire car elle donne aux 'frères' une certaine indépendance et peut les amener à favoriser leurs intérêts réciproques contre ceux de leurs parents consanguins ou affins.

[101] 12- Comme le remarquait Evans-Pritchard à propos de la fraternité de sang, il ne faut pourtant pas surestimer la force, souvent déclarée indestructible dans les entretiens, des parentés fictives. Du fait même qu'il s'agit de parentés aussi intéressées que les parentés ordinaires, elles ne sont pas non plus, comme elles, à l'abri de la découverte intéressée d'un intérêt supérieur. Comme je l'ai signalé dès 1977 pour les relations de parrainage à Karpathos, les relations qui liaient les bergers (qui ont le plus bas statut social) aux canacares (paysans les plus riches) et les rendaient fictivement 
Citation: Vernier, Bernard (2006) 'Du bon usage de la parenté construite avec des humeurs corporelles (sang et lait) et quelques autres moyens', European Journal of Turkish Studies, Thematic Issue $N^{\circ} 4$, The social practices of kinship. A comparative perspective, URL : http://www.ejts.org/document623.html

To quote a passage, use paragraph (§)

égaux, ne suffisaient pas à supprimer l'antagonisme des intérêts qui était au fondement même (il avait pour fonction le maintien de l'ordre social) de l'établissement de leur relation de parrainage : 'L'exemple des nombreux conflits passés démontre bien, à la fois, la réalité d'un antagonisme, toujours latent, entre les deux groupes, et le fait qu'il était, pour ainsi dire, à tel point structurellement inévitable que les relations de parrainage étaient incapables par elles seules de l'empêcher de se manifester. Mieux, l'exemple d'un canacare, président du village, attiré par son parrain berger dans un guet-apens qui lui coûta la vie, montre que les conflits étaient si violents qu'à l'occasion les relations de parrainage, loin de les atténuer, étaient elles-mêmes utilisées par l'une des familles qui y trouvait le meilleur moyen d'assouvir sa vengeance'(1977:180, 1991 : 81). II n'est pas étonnant qu'il en aille de même dans l'Hindu Kush et le Caucase chers à Parkes.

[102] Comme je l'ai montré (1977), les rapports de parenté sont prédisposés à enchanter, c'est-à-dire à occulter, à maintenir et à reproduire les rapports de domination et d'exploitation. C'est vrai pour la parenté consanguine (ex : les rapports de domination et d'exploitation aînés/cadettes à Karpathos, mais aussi les rapports aînés /cadets au Japon et dans le Béarn) et pour la parenté par alliance matrimoniale (les rapports hommes /femmes chez les Pomaques) C'est vrai aussi pour toutes les parentés fictives qui, quand elles s'établissent entre groupes sociaux inégaux (ou représentants de ces groupes), ont pour fonction la reproduction de l'ordre social ou, pour le dire autrement, la reproduction de la position de chaque groupe dans les rapports de force économique, symbolique ou politique. Entre eux, comme je l'ai montré pour le parrainage, la réciprocité est toujours inégale ou asymétrique. La violence symbolique exercée par les dominants n'est jamais assez efficace pour pouvoir exclure chez les dominés la conscience de cette asymétrie. C'est la raison pour laquelle l'amitié proclamée peut si facilement faire place à des actes d'hostilité. L'ambivalence des sentiments dont parle Parkes est l'indépassable de la relation. II faut d'autant plus proclamer la supériorité de la parenté fictive que personne n'est totalement dupe. C'est justement parce que la parenté de baptême est officiellement au-dessus de tout soupçon qu'on peut s'en servir pour tuer son filleul en l'attirant dans un guet-apens. Pour pouvoir progresser dans l'analyse, il faudrait pouvoir distinguer les parentés fictives et leur force différentielle selon la société. II est clair qu'on peut d'autant moins facilement transgresser les obligations ou les tabous qu'elles impliquent que la sanction automatique de la transgression est forte (la mort) et qu'il n'existe pas (contrairement 
Citation: Vernier, Bernard (2006) 'Du bon usage de la parenté construite avec des humeurs corporelles (sang et lait) et quelques autres moyens', European Journal of Turkish Studies, Thematic Issue $\mathrm{N}^{\circ} 4$, The social practices of kinship. A comparative perspective, URL : http://www.ejts.org/document623.html

To quote a passage, use paragraph (§)

à ce qui se passe dans les tribus du Nigéria) de rite permettant d'échapper à bon compte à une sanction pourtant présentée comme automatique.

[103] 13- Reste que les différentes parentés extraordinaires ont chacune leur spécificité qui demeure largement à explorer. La parenté de sang ne fournit que des frères, alors que la parenté de lait et la parenté baptismale donne des frères mais aussi des parents de lait. La différence de génération, comme dans le cas de la parenté consanguine, implique un respect tout spécifique de l'enfant pour ses parents de lait et singulièrement pour sa mère de lait. II faudrait probablement distinguer entre la parenté obtenu en faisant couler le sang lors d'un sacrifice (le rite d'intégration à la tribu décrit par Gélard) et la parenté par échange de sang. La parenté de sang peut abriter une alliance pour le mal (vol, assassinat) qui est exclue par la parenté baptismale.

[104] Comme l'ont bien vu les ethnologues, la parenté de lait donne parfois un pouvoir particulier aux femmes qui produisent cette humeur corporelle, un pouvoir que tend à remettre en cause, au moins dans le domaine des représentations, l'expression 'lait de l'étalon'.

[105] II semblerait que la parenté de lait vienne plus souvent redoubler la parenté consanguine et la relation d'affinité que la parenté de sang. C'est peut-être, en partie, que le lait est une substance organique nourricière qui peut, comme telle, entrer dans les échanges entre parents. On sait que l'islam a supprimé la fraternité de sang et a donné une importance particulière à la parenté de lait et que l'Eglise a parfois consacré la fraternité de sang et l'amitié jurée avant de l'interdire (cf.Fine). Reste à expliquer l'inégale répartition de la parenté de sang et de la parenté de lait dans le monde et à savoir si cela a un quelconque rapport avec la position de la femme dans les rapports de domination entre les sexes. Cette répartition semble en tout cas indépendante, à la fois de celle des systèmes de filiation et de celle des représentations sur la transmission des humeurs.

[106] 14- On aura pourtant compris qu'il n'y a pas pour nous d'institution spécifique liée à l'échange de sang. Cet échange n'est qu'une des multiples façons (citées plus haut) de créer des liens entre individus et/ou entre groupes.

[107] 15- Deux dangers guettent l'analyse de ces parentés que nous avons étudiées. Celui, bien représenté par le terme commode de 'parenté fictive', qui tend à en faire des parentés artificielles sans efficace sociale et celui, plus réel, de se laisser prendre au piège des métaphores (à caractère stratégique) utilisées par les 'indigènes' pour en faire l'équivalent approché de la parenté 
Citation: Vernier, Bernard (2006) 'Du bon usage de la parenté construite avec des humeurs corporelles (sang et lait) et quelques autres moyens', European Journal of Turkish Studies, Thematic Issue $\mathrm{N}^{\circ} 4$, The social practices of kinship. A comparative perspective, URL : http://www.ejts.org/document623.html

To quote a passage, use paragraph (§)

'consanguine'. L'ethnologue alors les surnaturalise, en tombant dans un substantialisme aveugle à l'existence de la 'mauvaise foi collective' et/ou à la prodigieuse capacité des sociétés à faire 'comme si'. II me semble qu'une anthropologie matérialiste des intérêts et des rapports de force permet mieux qu'une autre d'échapper à ces pièges. L'existence d'un interdit de mariage n'implique pas forcément l'existence d'une identité substantielle entre les personnes interdites, comme le prétendent certains ethnologues. Ajoutons pour finir que les hommes, en inventant la parenté de lait et la parenté de sang, utilisent une stratégie extrêmement courante pour légitimer l'ordre social en le fondant en nature. 

quelques autres moyens', European Journal of Turkish Studies, Thematic Issue $\mathrm{N}^{\circ} 4$, The social practices of kinship. A comparative perspective, URL : http://www.ejts.org/document623.html

To quote a passage, use paragraph $(\S)$

\section{References}

Altorki, Soraya (1980) 'Milk-kinship in Arab Society: An unexplored Problem in the Ethnography of Marriage', Ethnology 2 (XIX), pp. 233-244.

Beidelman, Thomas O. (1963) 'The Blood-Covenant and the Concept of Blood in Ukaguru', Africa XXXXIII, pp.321-342.

Biddulph, John (1880) Tribes of the Hindoo Koosh, Calcutta: Office of the superintendent of Printing (reprinted 1971, Gratz, Akademische Druck und Verlagsanstalt).

Bourdieu, Pierre (1972) Esquisse d'une théorie de la pratique, précédée de trois études kabyles, Genève, Droz.

Conte, Edouard (1994) 'Choisir ses parents dans la société arabe. La situation à l'avènement de l'islam', in Bonte Pierre (ed.) Epouser au plus proche. Inceste, prohibitions et stratégies matrimoniales autour de la Méditerranée, Paris, EHESS, pp. 149-187.

Corbier, Mireille (1999) 'La petite enfance à Rome: lois normes, pratiques individuelles et collectives', Annales 6, 1257-1290.

Dragadze, Tamara (1988) Rural Families in Soviet Georgia, Londres, Routledge.

Durham, M. Edith. (1909) High Albania, London, Allen and Unwin.

Eisenstadt, Shmuel Noah. (1956) 'Ritualized personnal Relations. Blood Brotherhood, best Friends, Compadre, etc., Man 96, pp.90-95.

Evans-Pritchard, Edward Evan (1933) 'Zande Blood Brotherhood', Africa VI, 4, pp.369-401.

Fay, Claude (1995) 'Car nous ne faisons qu'un.', Identités, équivalence, homologies en Maasina (Mali)', Cahiers des sciences humaines 31 (2), pp. 427-456.

Fine, Agnès (1994) Parrains marraines. La parenté spirituelle en Europe, Paris, Fayard.

Fortier, Corinne (2001) 'Le lait, le sperme, le dos. Et le sang ? Représentations physiologique de la filiation et de la parenté de lait en islam malékite et dans la société maure ?' Cahiers d'Etudes Africaines 161, http://etudesafricaines.revues.org/document68.html.

Gélard, Marie-Luce (2003) 'De la naissance au septième jour. Rituels féminins et temps suspendu (tribu berbérophone du Sud-Est marocain)', Ethnologie française XXXIII, 1.

Gélard, Marie-Luce (2004a) 'Modifications et ajustements événementiels des parentés électives dans le sud-Marocain', Awal 29.

Gélard, Marie-Luce (2004b) 'Protection par le lait dans la tribu des Aït Khebbach (Sud-Est Marocain)', Etudes Rurales 169-170, http://etudesrurales.revues.org/document2975.html.

Griaule, Marcel (1948) 'L'alliance cathartique', Africa IV (18), pp.242-258.

Hammel, E (1968) Alternative Social Structures and Ritual Relations in the Balkans, Englewood Cliffs, Prentice Hall. 
Citation: Vernier, Bernard (2006) 'Du bon usage de la parenté construite avec des humeurs corporelles (sang et lait) et quelques autres moyens', European Journal of Turkish Studies, Thematic Issue $N^{\circ} 4$, The social practices of kinship. A comparative perspective, URL : http://www.ejts.org/document623.html

To quote a passage, use paragraph $(\S)$

Hazoumé, Paul (1937) Le pacte de sang au Dahomey, Travaux et mémoires de L'institut d'ethnologie, Paris, XXV.

Hérodote, Histoires, Paris, Les Belles Lettres.

Jaussen, Antonin (1908) Coutumes arabes au pays de Moab, Paris, lib.Victor Lecoffre.

Jolly, Eric (1995) La bière du mil dans la société dogon, Thèse de doctorat, Université Paris X, Nanterre.

Khatib-Chahidi, Jane (1992) 'Milk kinship in Shi'ite Islamic Iran', in Maher V. (ed.), The Anthropology of Breast-Feeding, Oxford, Berg.

Klapisch-Zuber, Christiane (1990) La maison et le nom, Paris, Editions de l'EHESS.

Labouret, Henri (1929) 'La parenté à plaisanterie en Afrique occidentale', Africa II, pages ?

Leach, Edmund Ronald (1961) Rethinking Anthropology, Londres, University of London-Athlone.

Leitner, Gottlieb Wilhelm (1893) Dardistan in 1866, 1886 and 1893: being an account of the history, religions, customs, legends, fables, and songs of Gilgit, Chilas, Kandia (Gabrial), Yasin, Chitral, Hunza, Aagyr, and other parts of the Hindukush, New Delhi, Ausgabe Woking, Oriental University Institute.

Lévi-Strauss, Claude (1967), Les structures élémentaires de la parenté, Paris, Mouton.

Lyall, Sir Alfred (1882) Asiatic Studies Religious and Social, Londres, John Murray.

Magnarella, Paul; Turkdoğan, Orhan (1973) 'Descent, Affinity and Ritual Relations in Eastern Turkey', American Anthropologist LXXV (5), pp.1626-1633.

Malinowski, Bronislaw Kaspar (1931) 'Culture', Encyclopedia of the Social Sciences IV, New York, Macmillan, pp.621-646.

Malinowski, Bronislaw Kaspar (1967) La sexualité et sa répression dans les sociétés primitives, Paris, Payot.

Marcy, Georges (1936) 'L'alliance par colactation (tâd'a ) (en berbère). Chez les berbères du Maroc central', Revue africaine 79, pp. 957-973.

Meeks, C.K (1925) The Northern Tribes of Nigeria, Londres, Oxford University Press.

Mintz, Sydney ; Wolf, Eric. (1950) 'An Analysis of Ritual Co-Parenthood (Compadrazgo)', Southwestern Journal of Anthropology VI (4) pp.341-364.

Parkes, Peter (2001) 'Alternative Social Structures and Foster Relations in the Indu Kush: Milk Kinship Allegiance in Former Mountain Kingdoms of Northern Pakistan', Society for Comparative Study of Society and History, 43, pp. 4-36.

Parkes, Peter (2003) 'Fostering Fealty : A Comparative Analysis of Tributary Allegiances of Adoptive Kinship', Society for Comparative Study of Society and History, 45, pp. 741-782.

Parkes, Peter (2004) 'Fosterage, Kinship and Legend: When Milk Was Thicker than Blood ?', Society for Comparative Study of Society and History 46, pp. 587-615. 
Citation: Vernier, Bernard (2006) 'Du bon usage de la parenté construite avec des humeurs corporelles (sang et lait) et quelques autres moyens', European Journal of Turkish Studies, Thematic Issue $N^{\circ} 4$, The social practices of kinship. A comparative perspective, URL : http://www.ejts.org/document623.html

To quote a passage, use paragraph $(\S)$

Paul, B.D (1942) Ritual Kinship with Special Reference to Godparenthood in Middle America, PhD. thesis Univ. Chicago.

Paulme, Denise (1939) 'Parenté à plaisanterie et alliances par le sang en Afrique occidentale', Africa XII (4), pp. 433-444.

Paulme, Denise (1968) 'Pactes de sang, classes d'âge et castes en Afrique noire', Archives européennes de sociologie, 9 (1).

Seligman, Brenda (1935) 'The incest taboo as a social regulation' Sociological Review, vol 27, pp. 75-93.

Sertel, Ayşe Kudat. (1971) 'Ritual Kinship in Eastern Turkey', Anthropological Quartely, 44, pp.37-50.

Smith, William Robertson (1885) Kinship and Mariage inearly Arabia, Londres, Adam et Charles Black.

Smith, William Robertson (1914) Lectures on the religion of the Semites, Londres, Adam et Charles Black.

Tegnaeus, Harry (1954) La fraternité de sang, Paris, Payot.

Vernier, Bernard (1977) Rapports de parenté et rapports de domination, Thèse de troisième cycle dirigée par Pierre Bourdieu, Université René Descartes, Paris.

Vernier, Bernard (1980) 'La circulation des biens de la main d'œuvre et des prénoms à Karpathos : du bon usage des parents et de la parenté', Actes de la recherche en Sciences Sociales 31, pp.6387.

Vernier, Bernard (1984) 'Putting Kin and Kinship to Good Use : the Circulation of Goods, Labour, and Names on Karpathos (Greece)', in Medick, Hans. ; Sabean, David.Warren (eds.), Interest and Emotion, Cambridge, Cambridge University Press, Ed. MSH.

Vernier, Bernard (1991) La genèse sociale des sentiments. Aînés et cadets dans l'île grecque de Karpathos, Paris, Editions de l'EHESS.

Vernier, Bernard (1998), 'Représentation mythique du monde et domination masculine', Actes de le recherche en sciences sociales 125, pp.74-98.

Vernier, Bernard (1999), 'Du nouveau sur l'inceste? Pour une théorie unitaire', La Pensée 318, pp.53-80.

Vernier, Bernard (2005a) 'La prohibition de l'inceste dans le miroir des pactes de paix. A propos d'une enquête au Nigéria (région de Jos)', Regards sociologiques 30, pp.13-35.

Vernier, Bernard (2005b) 'Sociologie, ethnologie, un même combat scientifique', in Mauger Gérard (ed.), Rencontres avec Pierre Bourdieu, Bellecombe-en-Bauges, Editions du Croquant.

Vernier, Bernard (2005c) 'La prohibition des rapports sexuels et matrimoniaux avec les proches parents et alliés. Pour une théorie unitaire', Regards sociologiques 30, pp.37-60. 